\title{
Generalised permutation branes
}

\author{
Stefan Fredenhagen ${ }^{1}$ and Thomas Quella ${ }^{2}$ \\ ${ }^{1}$ Institut für Theoretische Physik, ETH-Hönggerberg, \\ CH-8093 Zürich, Switzerland \\ ${ }^{2}$ King's College London, Department of Mathematics, \\ Strand, London WC2R 2LS, United Kingdom
}

September 2005

\begin{abstract}
We propose a new class of non-factorising D-branes in the product group $G \times G$ where the fluxes and metrics on the two factors do not necessarily coincide. They generalise the maximally symmetric permutation branes which are known to exist when the fluxes agree, but break the symmetry down to the diagonal current algebra in the generic case. Evidence for the existence of these branes comes from a Lagrangian description for the open string world-sheet and from effective Dirac-Born-Infeld theory. We state the geometry, gauge fields and, in the case of $S U(2) \times S U(2)$, tensions and partial results on the open string spectrum. In the latter case the generalised permutation branes provide a natural and complete explanation for the charges predicted by K-theory including their torsion.
\end{abstract}




\section{Contents}

1 Introduction

2 Lagrangian description 3

2.1 Wess-Zumino-Novikov-Witten models . . . . . . . . . . . . . . . 3

2.2 Generalised permutation branes . . . . . . . . . . . . . . . . . . . 4

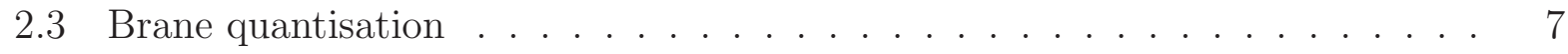

2.4 Discussion of gluing conditions . . . . . . . . . . . . . . 8

3 Generalised permutation branes in $S U(2) \times S U(2) \quad 9$

3.1 Self-intersections and charges . . . . . . . . . . . . . . . . . 9

3.2 Dirac-Born-Infeld analysis . . . . . . . . . . . . . . . . . . . 12

3.3 Brane tensions . . . . . . . . . . . . . . . . . . . . 15

3.4 Brane spectrum . . . . . . . . . . . . . . . . . . 17

4 Generalisation to higher rank groups 21

4.1 A useful parametrisation of simple groups . . . . . . . . . . . . . 22

4.2 Application to the product case . . . . . . . . . . . . . . . 23

5 Summary and outlook 25

$\begin{array}{ll}\text { A K-theory for products of groups } & 27\end{array}$

B Details of the Born-Infeld calculation 28

\section{Introduction}

Group manifolds have widely been used as a playground to study string theory in nontrivial, curved backgrounds. For compact groups, the conformal field theory (CFT) on the world-sheet is rational and completely under control. D-branes in these backgrounds have been investigated using boundary conformal field theory and semi-classical methods. It might surprise that despite all the progress that has been made in understanding branes on group manifolds, there are usually not enough D-branes known to explain the whole charge group predicted by (twisted) K-theory.

Soon after the essential role of (twisted) K-theory for the description of decay processes of D-branes was pointed out in [1, 2, 3, there have been attempts to explain the K-groups for group manifolds using boundary renormalisation group flow ideas. In this way the K-groups for $S U(2)$ and $S U(3)$ could be understood completely [4, 5, 6]. In the case of $S U(2)$ the only type of charge is associated with Cardy branes [7] while for $S U(3)$ there also exists a second type of charge which could be assigned to twisted branes [8]. Both classes of branes are maximally symmetric. Later the K-groups have been calculated in a purely mathematical manner [9, 10, 11]. According to these results the number of charges grows exponentially 
with the rank of the (simple) group $G$, but this exceeds the different types of maximally symmetric branes by far. Although there are some ideas what the branes carrying the remaining charges could be (see [12, 13] for a proposal for $S U(n)$ based on the symmetry breaking branes of [14, 15, 16]), it is fair to say that a satisfactory answer is still missing.

A simple example where one can hope to resolve the mismatch and explain the complete charge group is the Wess-Zumino-Novikov-Witten (WZNW) model on the product group $S U(2) \times S U(2)$. This theory has two parameters, the levels $k_{1}$ and $k_{2}$, which control the size and the H-field of the background. The corresponding K-theory is twisted by the third cohomology class $\tau=k_{1} \tau_{1}+k_{2} \tau_{2}$ where $\tau_{1}$ and $\tau_{2}$ are the generators of the third integer cohomology group of the two factors 1 It is given by (see appendix $\mathrm{A}$ )

$$
{ }^{\tau} K(S U(2) \times S U(2))=\mathbb{Z}_{k} \oplus \mathbb{Z}_{k}
$$

where $k=\operatorname{gcd}\left(k_{1}, k_{2}\right)$ is the greatest common divisor of the two levels. One summand in (11) belongs to 0-branes and can readily be explained by factorising Cardy branes; the charge relations derived from boundary renormalisation group flows as in [4, 5] produce the correct order $k$ of the charge group. The other summand corresponds to a three-dimensional cycle with vanishing integrated H-flux. When the two levels are equal, this cycle is wrapped by permutation branes [17] (see also [18, 19, 20] for more specific examples). The existence of the latter relies on an order- 2 automorphism of the affine Lie algebra $\widehat{s u}(2)_{k} \oplus \widehat{s u}(2)_{k}$ which interchanges the two factors. If the levels are different, no such automorphism exists, and there is no obvious CFT construction of D-branes corresponding to the second summand in the K-group. Let us nevertheless emphasise that we are in a special situation where the mismatch between K-theory and the known D-branes is cured for a particular choice of parameters $\left(k_{1}=k_{2}\right)$ and one can hope that this coincidence provides some hints regarding the appropriate construction of the missing branes for an arbitrary choice of the levels.

Following this reasoning we give a proposal for the branes that explain the missing charges. Instead of using the established constructions for symmetry breaking branes? we are directly led by the geometry of the branes that we expect from K-theory. We suggest the existence of "generalised permutation branes" in products $G \times G$ of Lie groups with different levels. The geometry of the simplest new brane is given by

$$
\left\{\left(g^{k_{2} / k}, g^{-k_{1} / k}\right) \mid g \in G\right\} \subset G \times G .
$$

It represents a $(\operatorname{dim} G)$-dimensional submanifold with vanishing total H-flux and reduces to the geometry of an ordinary permutation brane if $k_{1}=k_{2}$. This brane has higherdimensional cousins which are stabilised by a non-trivial F-field. They are described in section 2.2.

To support our proposal we derive the world-sheet Lagrangian and employ semi-classical target space methods (Dirac-Born-Infeld). The branes are not maximally symmetric, but

\footnotetext{
${ }^{1}$ Here, levels are understood as levels of the supersymmetric model.

${ }^{2}$ One could for instance break the $S U(2)$ symmetry down to the coset $S U(2) / U(1)$ and $U(1)$ and then perform a twist in the $U(1)$ part, but the corresponding branes seem to have the wrong topology to explain the charges.
} 
the symmetry of the diagonally embedded group $G$ is still conserved. For given levels, there are only finitely many generalised permutation branes. Their number coincides with the number of untwisted maximally symmetric branes in a single group factor $G$ with level $\kappa=\operatorname{lcm}\left(k_{1}, k_{2}\right)$, the least common multiple of $k_{1}$ and $k_{2}$. In the case of $S U(2) \times S U(2)$ we also analyse tensions and charges of the new branes. The relative tensions are controlled by the modular S-matrix of a single $S U(2)$-model with level $\kappa$. Stability considerations indicate that the charge of the branes is conserved modulo $k=\operatorname{gcd}\left(k_{1}, k_{2}\right)$ which is in perfect agreement with the K-theory result.

The plan of the paper is as follows. In section 2 we discuss the $\sigma$-model on a worldsheet with boundaries. After a short introduction into WZNW models, we formulate the geometry of our proposed generalised permutation branes in the product group $G \times G$ and give an expression for the boundary two-form living on them. Then the quantisation condition which leads to a discrete brane spectrum is discussed and it is shown that the branes preserve the diagonal $G$-symmetry.

Section 3 concentrates on the case of $S U(2) \times S U(2)$. First we analyse the geometry of the branes in detail and show that only a subset of the world volumes avoid self-intersections and can be considered stable. The instability of the other branes reproduces the expected charge group including its torsion. In the following we discuss the effective Dirac-Born-Infeld description. It is shown that the proposed branes satisfy the equations of motions, partly using numerical methods. Also the tensions and the open string spectrum are analysed numerically.

Section 4 generalises some of the results of section 3 to higher rank groups. A summary and a discussion of open problems and future directions conclude the paper. In particular, we comment on a possible extension of our results to cosets where generalised permutation branes recently appeared in the rather complementary approach of matrix factorisations in the topological subsector of products of $N=2$ minimal models [21]. Two appendices contain the computation of the relevant K-groups and the technical details of the DBI calculations.

\section{Lagrangian description}

\subsection{Wess-Zumino-Novikov-Witten models}

The propagation of strings on a group manifold $\mathcal{G}$ is described by a WZNW model [22, 23], a special and conformally invariant instance of non-linear $\sigma$-models. Since we are interested in open strings, the world-sheet $\Sigma$ has a non-trivial boundary. For our purposes it is sufficient to assume that $\partial \Sigma$ consists of precisely one component with the topology of a circle. At this boundary, the group-valued field $g$ on $\Sigma$ is constrained to some subset $\mathcal{D} \subset \mathcal{G}$, the D-brane. The action reads [24, 25]

$$
\mathcal{S}^{\mathcal{G}}[g]=\frac{1}{4 \pi i} \int_{\Sigma}\left\langle g^{-1} \partial g, g^{-1} \bar{\partial} g\right\rangle d z \wedge d \bar{z}+\frac{1}{4 \pi i} \int_{B} \omega^{\mathrm{WZ}}-\frac{1}{4 \pi i} \int_{D} \omega_{C} .
$$


Here, $\langle\cdot, \cdot\rangle$ is a non-degenerate invariant bilinear form on the associated Lie algebra, and the two-dimensional disc $D$ is used to fill the hole in $\Sigma$, such that $\Sigma \cup D$ has no boundary. As usual the Wess-Zumino term is defined as an integral of the Wess-Zumino form

$$
\omega^{\mathrm{WZ}}(g)=\frac{1}{6}\left\langle g^{-1} d g,\left[g^{-1} d g, g^{-1} d g\right]\right\rangle
$$

over a three-dimensional space $B$ with $\partial B=\Sigma \cup D$. The theory should not depend on the choice of the two auxiliary manifolds $D$ and $B$ and the continuation of the field $g$ to $D$ and $B$. This requires the Wess-Zumino form to be integral and trivialised by the boundary two-form on the brane $\mathcal{D}$,

$$
\left.\omega^{\mathrm{WZ}}\right|_{\mathcal{D}}=d \omega_{C}
$$

The discussion of a further quantisation condition for $\omega_{C}$ will be postponed until section 2.3. If all these constraints are satisfied, the action (3) is well-defined up to multiples of $2 \pi i$ which do not affect the path integral. We refer to the literature for details.

In this paper we are interested in string theory on the product group $\mathcal{G}=G \times G$ where $G$ is a compact simply-connected simple group. If we denote by ' $\operatorname{tr}$ ' the suitably normalised Killing form on $\mathfrak{g}=\operatorname{Lie}(G)$, then the most general non-degenerate invariant bilinear form on the product is given by

$$
\left\langle\left(X_{1}, X_{2}\right),\left(Y_{1}, Y_{2}\right)\right\rangle=k_{1} \operatorname{tr}\left(X_{1} Y_{1}\right)+k_{2} \operatorname{tr}\left(X_{2} Y_{2}\right)
$$

with non-vanishing real numbers $k_{i}$. The integrality of the Wess-Zumino form and unitarity of the (Minkowskian version of the) theory imposes the constraint that the levels $k_{i}$ are non-negative integers.

\subsection{Generalised permutation branes}

In each group $\mathcal{G}$ there exist so-called maximally symmetric branes which are associated to an automorphism $\Omega$ of $\mathcal{G}$ provided the latter may be lifted to the underlying affine Lie algebra of the WZNW model. On the CFT side these branes have been constructed in [7, 8]. On the geometric side they have later been shown to wrap twisted conjugacy classes [24, 26]

$$
\mathcal{C}_{\Omega}(f)=\left\{g f \Omega\left(g^{-1}\right) \mid g \in \mathcal{G}\right\} .
$$

These submanifolds admit an obvious action of $\mathcal{G}$ which corresponds to the affine symmetry preserved in the boundary CFT.

Let us now focus on the product group $\mathcal{G}=G \times G$. It can easily be understood that there are maximally symmetric branes which completely factorise. They correspond to automorphisms which may be written as a product of automorphisms. On the other hand the group $\mathcal{G}$ also admits the permutation automorphism

$$
\tau\left(g_{1}, g_{2}\right)=\left(g_{2}, g_{1}\right) .
$$


The associated twisted conjugacy classes have the form

$$
\mathcal{C}_{\tau}(f)=\left\{\left(h_{1} f h_{2}^{-1}, h_{2} f h_{1}^{-1}\right) \mid h_{1}, h_{2} \in G\right\}
$$

and one might believe that they are good candidates for branes. This assertion is certainly true for $k_{1}=k_{2}$. Whenever the two levels disagree, however, the permutation automorphism $\tau$ does not leave the metric invariant and cannot be lifted to an automorphism of the affine Lie algebra $\hat{\mathfrak{g}}_{k_{1}} \oplus \hat{\mathfrak{g}}_{k_{2}}$ which underlies the WZNW model. We thus conclude that the twisted conjugacy classes (9) just describe the loci of branes if $k_{1}=k_{2} 3$

The main result of our paper is that a slight modification of the geometry (9) actually gives rise to proper D-branes even for $k_{1} \neq k_{2}$. To be more concrete, we propose that the submanifold

$$
\mathcal{D}_{\tau}(f)=\left\{\left(\left(h_{1} f h_{2}^{-1}\right)^{k_{2}^{\prime}},\left(h_{2} f h_{1}^{-1}\right)^{k_{1}^{\prime}}\right) \mid h_{1}, h_{2} \in G\right\},
$$

where $k_{i}^{\prime}=k_{i} / k$ and $k=\operatorname{gcd}\left(k_{1}, k_{2}\right)$, is the locus of a generalised permutation brane if we impose suitable quantisation conditions on the constant $f$ which may be chosen from a fixed maximal torus $T \subset G$. Obviously, this geometry reduces to the twisted conjugacy class (9) whenever the two levels coincide. In general the dimension of the branes is ( $2 \operatorname{dim} G-\operatorname{rank} G$ ) but for certain degenerate values of $f$ it will have a lower value. In particular if $f$ equals the identity element $e \in G$, the expression (10) simplifies to

$$
\mathcal{D}_{\tau}(e)=\left\{\left(g^{k_{2}^{\prime}}, g^{-k_{1}^{\prime}}\right) \mid g \in G\right\}
$$

In this case the dimension is given by $\operatorname{dim} G$. Since the constants $k_{i}^{\prime}$ are relatively prime, there is a one-to-one correspondence between elements of $\mathcal{D}_{\tau}(e)$ and elements of $G$.

The reason for the occurrence of the non-trivial exponents $k_{1}^{\prime}$ and $k_{2}^{\prime}$ in the definition (10) becomes immediately obvious if we remember that we have to find a boundary twoform $\omega_{C}$ which trivialises the Wess-Zumino form on the brane. Repeatedly applying the Polyakov-Wiegmann identity

$$
\omega^{\mathrm{WZ}}(h g)=\omega^{\mathrm{WZ}}(h)+\omega^{\mathrm{WZ}}(g)-d \operatorname{tr}\left(h^{-1} d h \wedge d g g^{-1}\right)
$$

one can easily prove

$$
\omega^{\mathrm{WZ}}\left(g^{n}\right)=n \omega^{\mathrm{WZ}}(g)+\sum_{j=1}^{n-1}(n-j) d \operatorname{tr}\left(\operatorname{Ad}_{g}^{j}\left(g^{-1} d g\right) \wedge g^{-1} d g\right) .
$$

Here, $\operatorname{Ad}_{g}(X)=g X g^{-1}$ denotes the adjoint action of the group element $g$ on $X \in \mathfrak{g}$. If we instead consider the total Wess-Zumino form $\omega^{\mathrm{WZ}}\left(g_{1}, g_{2}\right)=k_{1} \omega^{\mathrm{WZ}}\left(g_{1}\right)+k_{2} \omega^{\mathrm{WZ}}\left(g_{2}\right)$ and restrict it to the submanifold (10), one realises that the constants $k_{i}^{\prime}$ have been chosen in

\footnotetext{
${ }^{3}$ Reading [27] one might get the impression that all twisted conjugacy classes are loci of D-branes. In their analysis, however, the authors implicitly assumed that the automorphism preserves the scalar product. For simple groups all automorphisms possess this property.

${ }^{4}$ This proposal came up in discussions with A. Alekseev.
} 
a way such that the terms which cannot be written as a total derivative cancel out. As a consequence we find that

$$
\left.\omega^{\mathrm{WZ}}\right|_{\mathcal{D}_{\tau}(f)}=d \omega_{C}
$$

is satisfied if we choose the boundary two-form $\omega_{C}$ according to

$$
\begin{gathered}
\omega_{C}=\frac{k_{1} k_{2}}{k}\left\{\operatorname{tr}\left(h_{1}^{-1} d h_{1} \wedge \operatorname{Ad}_{f}\left(h_{2}^{-1} d h_{2}\right)\right)+\operatorname{tr}\left(h_{2}^{-1} d h_{2} \wedge \operatorname{Ad}_{f}\left(h_{1}^{-1} d h_{1}\right)\right)\right\} \\
+k_{1} \sum_{j=1}^{k_{2}^{\prime}-1}\left(k_{2}^{\prime}-j\right) \operatorname{tr}\left(\operatorname{Ad}_{g^{j}}\left(g^{-1} d g\right) \wedge g^{-1} d g\right)_{g=h_{1} f h_{2}^{-1}} \\
+k_{2} \sum_{j=1}^{k_{1}^{\prime}-1}\left(k_{1}^{\prime}-j\right) \operatorname{tr}\left(\operatorname{Ad}_{g^{j}}\left(g^{-1} d g\right) \wedge g^{-1} d g\right)_{g=h_{2} f h_{1}^{-1}} .
\end{gathered}
$$

Note that the first two terms compensate each other if $f$ is central. If in addition the two levels coincide, the boundary two-form vanishes identically.

Let us finally comment on the symmetries of our generalised permutation branes. We already mentioned that the branes are maximally symmetric in the case of equal levels $k_{1}=k_{2}=k$, i.e. they preserve the current algebra $\hat{\mathfrak{g}}_{k} \oplus \hat{\mathfrak{g}}_{k}$. On the geometric side this corresponds to the invariance of the brane world volume (9) under the two different twisted adjoint actions

$$
\left(g_{1}, g_{2}\right) \mapsto\left(h g_{1}, g_{2} h^{-1}\right) \quad \text { and } \quad\left(g_{1}, g_{2}\right) \mapsto\left(g_{1} h^{-1}, h g_{2}\right)
$$

For $k_{1} \neq k_{2}$, however, the world volumes (10) and (11) are not invariant anymore under this action of $G \times G$ because of the non-trivial exponents $k_{i}^{\prime}$. Instead they only admit an action of the diagonal subgroup

$$
\left(g_{1}, g_{2}\right) \mapsto\left(h g_{1} h^{-1}, h g_{2} h^{-1}\right)
$$

in that case. We thus expect that the affine symmetry is at least broken down to $\hat{\mathfrak{g}}_{k_{1}+k_{2}}$. In fact we will argue in section 2.4 that this smaller symmetry is indeed conserved 5

We conclude that generalised permutation branes provide a new, geometrically motivated, class of symmetry breaking D-branes. In particular we wish to emphasise that the non-maximally symmetric branes constructed in [15, 16] have a geometrical interpretation which is very distinct from (10). In addition, those branes also exist for $k_{1}=k_{2}$ and certainly do not coincide with ordinary permutation branes in that case.

\footnotetext{
${ }^{5}$ Note that in the CFT description the branes have to preserve the Virasoro algebra associated with $\hat{\mathfrak{g}}_{k_{1}} \oplus \hat{\mathfrak{g}}_{k_{2}}$. Since the central charge of the diagonal current algebra $\hat{\mathfrak{g}}_{k_{1}+k_{2}}$ is not sufficient, there also has to be an additional symmetry including a Virasoro algebra with the remaining central charge. So far we could not identify this additional symmetry but according to our geometric arguments it will quite certainly not be of affine type.
} 


\subsection{Brane quantisation}

In the previous section we described the geometry of generalised permutation branes. The branes are labelled by an element $f$ of the Cartan torus of $G$. As always for branes in WZNW models, there is a quantisation condition which restricts the possible values of $f$ to a discrete set. It arises from an ambiguity in the Lagrangian description whenever the brane geometry has a non-trivial two-cycle (see e.g. [28, 24, 25]). Namely, the action $\mathcal{S}^{\mathcal{G}}$ in (3) depends on how the map $g$ is continued from $\Sigma$ to the whole $\Sigma \cup D$. The ambiguity takes the form

$$
\Delta \mathcal{S}^{\mathcal{G}}=\frac{1}{4 \pi i}\left(\int_{D_{3}} \omega^{\mathrm{WZ}}-\int_{S^{2}} \omega_{C}\right)
$$

for a three dimensional ball $D_{3}$, whose boundary $S^{2}$ is mapped into the brane. To make the path integral well defined, $\Delta \mathcal{S}^{\mathcal{G}}$ has to be a multiple of $2 \pi i$. In other words, the F-field $F=\omega_{C}-B$ (where $B$ is a two-form such that $d B=\omega^{\mathrm{WZ}}$ ) must have quantised flux through any two-sphere embedded into the brane [29].

In [25, 27] the quantisation condition has been worked out for untwisted branes in arbitrary compact simple simply-connected Lie groups $G$. The allowed branes are localised on conjugacy classes $\mathcal{C}(t) \subset G$ of elements

$$
t=\exp \frac{2 \pi i \lambda}{k}
$$

in the Cartan torus where $\lambda$ is an integral weight of $\mathfrak{g}$.

The generalised permutation branes $\mathcal{D}_{\tau}(f)$ in the product group are described by an immersion of $G \times \mathcal{C}\left(f^{2}\right)$ into $G \times G$,

$$
G \times \mathcal{C}\left(f^{2}\right) \ni(g, c) \mapsto\left(g^{k_{2}^{\prime}},\left(c g^{-1}\right)^{k_{1}^{\prime}}\right) \in \mathcal{D}_{\tau}(f) .
$$

Ignoring possible self-intersections (see section 3.1), the only non-trivial two-cycles in the brane come from the conjugacy class $\mathcal{C}\left(f^{2}\right)$. So we can restrict our analysis to the submanifold $\{e\} \times \mathcal{C}\left(f^{2}\right)$ which is embedded in $G \times G$ as $\{e\} \times\left(\mathcal{C}\left(f^{2}\right)\right)^{k_{1}^{\prime}}=\{e\} \times \mathcal{C}\left(f^{2 k_{1}^{\prime}}\right)$. On this conjugacy class the boundary two-form $\omega_{C}$ restricts to the usual two-form of untwisted branes in a single copy of $G$ with level $k_{2}$. Employing the result (19), we find that $t=f^{2 k_{1}^{\prime}}$ can take the quantised values $\exp \frac{2 \pi i \lambda}{k_{2}}$, i.e. $f$ is restricted to

$$
f=\exp \frac{\pi i \lambda}{\kappa}
$$

where $\kappa=k k_{1}^{\prime} k_{2}^{\prime}$ is the least common multiple of the levels $k_{1}$ and $k_{2}$.

The number of generalised permutation branes thus coincides with the number of untwisted branes in a single copy of $G$ with level $\kappa$. Surprisingly enough this suggests that the exact CFT description will be based on a rational conformal subalgebra of the bulk symmetry $\hat{\mathfrak{g}}_{k_{1}} \oplus \hat{\mathfrak{g}}_{k_{2}}$ and gives a severe restriction on the complete set of symmetries of the brane. 


\subsection{Discussion of gluing conditions}

To better understand the new branes, we would like to inspect the gluing conditions on the boundary. Let us assume that $\Sigma$ is the upper half-plane. The conserved currents of the bulk-model are

$$
J_{j}(z)=-k_{j} \partial g_{j} g_{j}^{-1}=-\frac{k_{j}}{2} \partial_{x} g_{j} g_{j}^{-1}+\frac{i k_{j}}{2} \partial_{y} g_{j} g_{j}^{-1}
$$

and

$$
\bar{J}_{j}(z)=k_{j} g_{j}^{-1} \bar{\partial} g_{j}=\frac{k_{j}}{2} g_{j}^{-1} \partial_{x} g_{j}+\frac{i k_{j}}{2} g_{j}^{-1} \partial_{y} g_{j},
$$

where $g_{1}(z, \bar{z})$ and $g_{2}(z, \bar{z})$ are the bulk fields. At the boundary, the currents $J_{j}$ and $\bar{J}_{j}$ are related. We will show in the following that the diagonal combination $J_{1}+J_{2}$ equals the anti-holomorphic counterpart $\bar{J}_{1}+\bar{J}_{2}$ at $z=\bar{z}$.

From the brane geometry (10), we find a condition on $\partial_{x} g_{i}$ : for $y=0$ we have $g_{1}=$ $\left(h_{1} f h_{2}^{-1}\right)^{k_{2}^{\prime}}$ and $g_{2}=\left(h_{2} f h_{1}^{-1}\right)^{k_{1}^{\prime}}$, hence

$$
\begin{aligned}
g_{1}^{-1} \partial_{x} g_{1} & =\frac{1-\operatorname{Ad}_{g_{1}}^{-1}}{1-\operatorname{Ad}_{h_{1} f h_{2}^{-1}}^{-1}}\left(\left(h_{1} f h_{2}^{-1}\right)^{-1} \partial_{x}\left(h_{1} f h_{2}^{-1}\right)\right) \\
g_{2}^{-1} \partial_{x} g_{2} & =\frac{1-\mathrm{Ad}_{g_{2}}^{-1}}{1-\mathrm{Ad}_{h_{2} f h_{1}^{-1}}^{-1}}\left(\left(h_{2} f h_{1}^{-1}\right)^{-1} \partial_{x}\left(h_{2} f h_{1}^{-1}\right)\right) .
\end{aligned}
$$

This provides a relation between $g_{1}^{-1} \partial_{x} g_{1}$ and $g_{2}^{-1} \partial_{x} g_{2}$ at the boundary.

For a further relation we have to study the equations of motion. We vary the action and restrict our attention to the contribution from the boundary. From the kinetic term we find

$$
\left.\delta \mathcal{S}_{\text {kin }}^{\mathcal{G}}\right|_{\text {boundary }}=\frac{k_{1}}{4 \pi} \int d x \operatorname{tr} g_{1}^{-1} \delta g_{1} g_{1}^{-1} \partial_{y} g_{1}+\frac{k_{2}}{4 \pi} \int d x \operatorname{tr} g_{2}^{-1} \delta g_{2} g_{2}^{-1} \partial_{y} g_{2}
$$

The variations of $g_{1}$ and $g_{2}$ on the boundary are related, because $g_{1}=\left(h_{1} f h_{2}^{-1}\right)^{k_{2}^{\prime}}$ and $g_{2}=\left(h_{2} f h_{1}^{-1}\right)^{k_{1}^{\prime}}$. For our purpose we do not need the full boundary equations of motion, so we concentrate on variations such that

$$
\delta h_{1} h_{1}^{-1}=\delta h_{2} h_{2}^{-1}=\delta h h^{-1} .
$$

Under this assumption we obtain

$$
\left.\delta \mathcal{S}_{\text {kin }}^{\mathcal{G}}\right|_{\text {boundary }}=-\frac{1}{4 \pi} \int d x \operatorname{tr}\left(\delta h h^{-1}\right)\left[k_{1}\left(1-\operatorname{Ad}_{g_{1}}\right)\left(g_{1}^{-1} \partial_{y} g\right)+k_{2}\left(1-\operatorname{Ad}_{g_{2}}\right)\left(g_{2}^{-1} \partial_{y} g_{2}\right)\right] \text {. }
$$

There is another contribution to $\delta S$ of that form which comes from the integral over the boundary two-form $\omega_{C}$ given in (15). We find

$$
\begin{aligned}
\left.\delta \mathcal{S}_{\mathrm{WZ}}^{\mathcal{G}}\right|_{\text {boundary }} & =\frac{i}{4 \pi} \int d x \operatorname{tr}\left(\delta h h^{-1}\right)\left\{-k_{1} k_{2}^{\prime}\left[\operatorname{Ad}_{h_{1} f}\left(h_{2}^{-1} \partial_{x} h_{2}\right)-\operatorname{Ad}_{h_{2} f^{-1}}\left(h_{1}^{-1} \partial_{x} h_{1}\right)\right]\right. \\
+ & \left.\left(1-\operatorname{Ad}_{g}\right) \sum_{j=1}^{k_{2}^{\prime}-1} k_{1}\left(k_{2}^{\prime}-j\right)\left(\operatorname{Ad}_{g}^{-j}-\operatorname{Ad}_{g}^{j}\right)\left(g^{-1} \partial_{x} g\right)\right\}_{g=h_{1} f h_{2}^{-1}}+(1 \leftrightarrow 2) .
\end{aligned}
$$


This leads to the equation of motion

$$
\begin{aligned}
& k_{1}\left(1-\operatorname{Ad}_{g_{1}}\right)\left(g_{1}^{-1} \partial_{y} g\right)+k_{2}\left(1-\operatorname{Ad}_{g_{2}}\right)\left(g_{2}^{-1} \partial_{y} g_{2}\right) \\
&=\left\{-i k_{1} k_{2}^{\prime}\left[\operatorname{Ad}_{h_{1} f}\left(h_{2}^{-1} \partial_{x} h_{2}\right)-\operatorname{Ad}_{h_{2} f^{-1}}\left(h_{1}^{-1} \partial_{x} h_{1}\right)\right]-i k_{1} k_{2}^{\prime}\left(1+\operatorname{Ad}_{g}\right)\left(g^{-1} \partial_{x} g\right)\right. \\
&\left.\quad+i k_{1}\left(1+\operatorname{Ad}_{g_{1}}\right) \frac{1-\operatorname{Ad}_{g_{1}}^{-1}}{1-\operatorname{Ad}_{g}}\left(g^{-1} \partial_{x} g\right)\right\}_{g=h_{1} f h_{2}^{-1}}+(1 \leftrightarrow 2) .
\end{aligned}
$$

Using the relations (22) from the brane geometry we obtain

$$
k_{1}\left(1-\operatorname{Ad}_{g_{1}}\right)\left(g_{1}^{-1} \partial_{y} g_{1}\right)+(1 \leftrightarrow 2)=i k_{1}\left(1+\operatorname{Ad}_{g_{1}}\right)\left(g_{1}^{-1} \partial_{x} g_{1}\right)+(1 \leftrightarrow 2) .
$$

Expressing the result in terms of the currents $J_{j}$ and $\bar{J}_{j}$ we finally arrive at the desired boundary condition

$$
J_{1}+J_{2}=\bar{J}_{1}+\bar{J}_{2}
$$

We expect that the associated symmetry of the classical boundary theory continues to hold in the full quantum theory.

\section{Generalised permutation branes in $S U(2) \times S U(2)$}

In this section we will analyse some of the properties of our generalised permutation branes (10). For technical reasons we restrict ourselves to the simplest and physically most interesting case, the product group $S U(2)_{k_{1}} \times S U(2)_{k_{2}}$. First we present a detailed discussion of the geometry of the branes. We argue that the occurrence of self-intersections for certain values of the brane labels causes instabilities and allows us to predict the torsion of the brane charges. Afterwards we provide additional support for the existence of the new branes using the Dirac-Born-Infeld approach. Finally, the tensions of the branes and their low-energy excitations are discussed.

\subsection{Self-intersections and charges}

Let us take a closer look at the proposed brane geometry. For the simplest brane, the map

$$
G \rightarrow G \times G: \quad g \mapsto\left(g^{k_{2}^{\prime}}, g^{-k_{1}^{\prime}}\right)
$$

provides a smooth embedding of $G$ into $G \times G$. Note that the map is one-to-one: from $g_{1}=g^{k_{2}^{\prime}}$ and $g_{2}=g^{-k_{1}^{\prime}}$ one can reproduce $g$ by taking the product of appropriate powers of $g_{1}$ and $g_{2}\left(k_{1}^{\prime}\right.$ and $k_{2}^{\prime}$ are coprime). For the higher dimensional branes, we have a parametrisation by the map (20) from $G \times \mathcal{C}\left(f^{2}\right)$ into $G \times G$ where $\mathcal{C}\left(f^{2}\right) \subset G$ is the conjugacy class of $f^{2}$ in $G$. As long as the element $f$ of the Cartan torus is close to the group unit, the situation is very similar to the case of the simplest brane, and the map is still one-to-one. For larger conjugacy classes $\mathcal{C}\left(f^{2}\right)$, however, the map ceases to be one-to-one and the brane develops self-intersections. This signals an instability of the brane. In the following we 


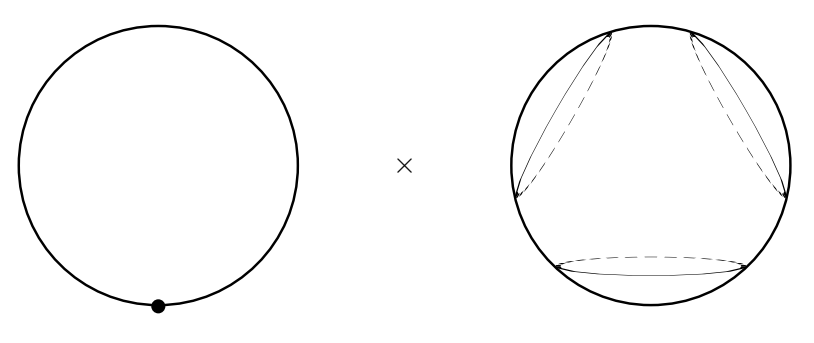

Figure 1: The intersection of $\mathcal{D}_{\tau}^{\text {red }}(f)$ with the slice $\{e\} \times S U(2)$ for "reduced" levels $k_{1}^{\prime}=1$ and $k_{2}^{\prime}=3$ : The group manifold of $S U(2)$ which is a three-sphere $S^{3}$ is drawn as a twosphere, the circles represent spherical conjugacy classes.

will take $S U(2) \times S U(2)$ as an example and work out the precise conditions under which intersections occur and what the instabilities imply for the torsion of the brane charges.

The brane geometry is invariant under the adjoint action of the diagonally embedded $S U(2)$. This action cannot produce any self-intersections, so we can restrict our discussion to a generating set of the full orbit which can be chosen to be

$$
\mathcal{D}_{\tau}^{\text {red }}(f)=\left\{\left(t^{k_{2}^{\prime}},\left(c t^{-1}\right)^{k_{1}^{\prime}}\right) \mid t \in T, c \in \mathcal{C}\left(f^{2}\right)\right\}
$$

Let us for a moment restrict to a simple case and assume that $k_{1}^{\prime}=1$. To get an idea of the geometry we look at the slice $\{e\} \times S U(2) \subset S U(2) \times S U(2)$. What is the intersection of $\mathcal{D}_{\tau}^{\text {red }}(f)$ with this slice? The equation $t^{k_{2}^{\prime}}=e$ has $k_{2}^{\prime}$ solutions, namely

$$
t_{j}=\left(\begin{array}{cc}
e^{\frac{2 \pi i j}{k_{2}^{\prime}}} & 0 \\
0 & e^{-\frac{2 \pi i j}{k_{2}^{\prime}}}
\end{array}\right) \quad j=0, \ldots, k_{2}^{\prime}-1 .
$$

The restriction of $\mathcal{D}_{\tau}^{\text {red }}(f)$ to the slice is the superposition of $k_{2}^{\prime}$ copies of the conjugacy class $\mathcal{C}\left(f^{2}\right)$ where each copy is translated by $t_{j}^{-1}$. In figure 1 the resulting geometry is illustrated for $k_{2}^{\prime}=3$.

We parametrise $f$ by

$$
f^{2}=\left(\begin{array}{cc}
e^{i \xi} & 0 \\
0 & e^{-i \xi}
\end{array}\right)
$$

One can immediately see that self-intersections will occur when the angle $\xi$ reaches the critical value $\xi^{c}=\frac{\pi}{k_{2}^{\prime}}$. Figures 2 and 3 show the geometry for $\xi$ at and above the critical angle.

The geometry of the brane at the critical angle does not have any non-trivial two-cycle, so there is no F-flux that could stabilise the brane: the brane will decay. Above the critical angle, the intersections shown in figure 3 destabilise the brane. One might expect that the brane dissolves at the intersections and decays into a brane with an angle $\xi$ below the critical one (see figure 4). 


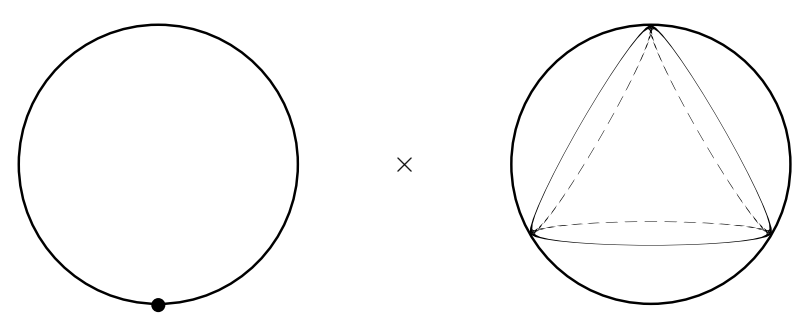

Figure 2: The brane geometry at the critical angle.

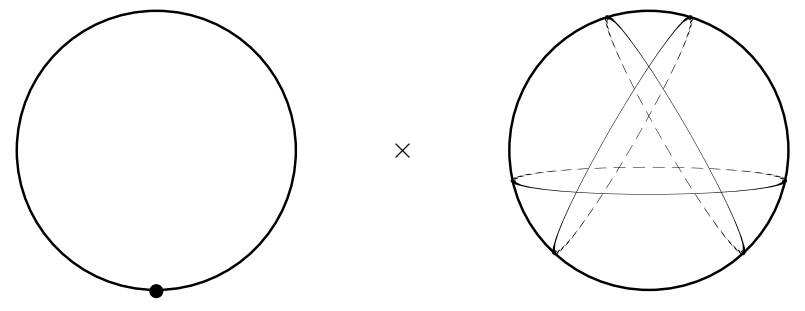

Figure 3: The brane geometry above the critical angle.

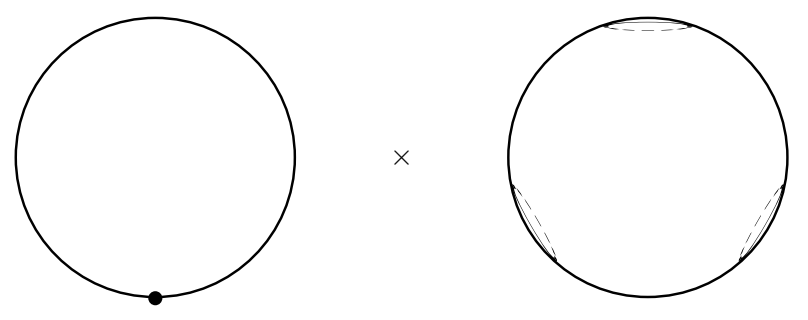

Figure 4: The brane of figure 3 might decay into this brane of lower angle (which is obtained by translation in the second factor of $S U(2) \times S U(2))$. 
The analysis can easily be extended to the case of arbitrary levels. The critical angle is then given by

$$
\xi^{c}=\frac{\pi}{k_{1}^{\prime} k_{2}^{\prime}}
$$

What can we learn about brane charges? By comparison with ordinary untwisted branes in $S U(2)$, it is suggestive to take the F-flux as counting the charge. Then the first brane at angle $\xi_{1}=\pi / \kappa$ carries charge 1 , the second brane charge 2 and so on. The $k^{\text {th }}$ brane sits at the critical angle, $\xi_{k}=\xi^{c}=\pi \frac{k}{\kappa}$, and it should have charge zero. This means that the brane charge takes values in the group $\mathbb{Z}_{k}$ and there are $k-1$ branes without self-intersections with angles $\xi_{n}=\pi \frac{n}{\kappa}$ where $n=1, \ldots, k-1$. This is precisely the charge group which we expected from K-theory, confirming that the proposed branes carry the missing K-theory charges.

\subsection{Dirac-Born-Infeld analysis}

\subsubsection{Preliminaries}

In the geometric limit of string theory the dynamics of D-branes is known to be described by the Dirac-Born-Infeld (DBI) action [30, 31, 32]. In our situation where the dilaton is constant, the action functional may be written 6

$$
\mathcal{S}_{\mathrm{DBI}} \sim \int_{\mathcal{D}} d^{p} y \sqrt{\operatorname{det}(\hat{g}+\hat{B}+F)} .
$$

The integral is performed over the $p$-dimensional world volume of the D-brane and $\hat{g}$ and $\hat{B}$ are the induced metric and the induced B-field, respectively. These two terms depend on the embedding of the brane into the given string background. In addition we have a two-form field $F$ which lives on the brane. The fields $\hat{B}$ and $F$ are not gauge invariant but their sum is and it agrees with the boundary two-form $\omega_{C}$ (15) that we found in the Lagrangian setting. The DBI approach should be applicable to our generalised permutation branes in the limit of large levels. Since the geometry heavily depends on the coprime integers $k_{1}^{\prime}$ and $k_{2}^{\prime}$, the latter should be kept fixed in that limit.

The Dirac-Born-Infeld action allows us to extract important information about the properties of D-branes. First and most important it tells us which submanifolds are allowed as D-branes. The latter correspond to embeddings of the $p$-dimensional world volume into the target space which minimise the DBI action. By calculating the fluctuations about such a solution we can also gain knowledge about the low-energy excitations of the brane. In the case of maximally symmetric branes in compact group manifolds this program has been carried out in [29, 27]. In this section we want to show that our proposal (10) for the geometry of generalised permutation branes indeed minimises the functional (33), thus proving conformal invariance.

\footnotetext{
${ }^{6}$ We assume Neumann boundary conditions in the time direction and ignore the latter from now on.
} 
The variation of the brane embedding leads to the gauge invariant equations of motion

$$
\left[\left(\hat{g}+\omega_{C}\right)^{-1}\right]^{b a} \Omega_{a b}^{\mu}=0
$$

which have been derived in 33 . This has to be supplemented by the condition

$$
\partial_{b}\left(\sqrt{\operatorname{det}\left(\hat{g}+\omega_{C}\right)}\left[\left(\hat{g}+\omega_{C}\right)^{-1}\right]_{\text {antisym }}^{a b}\right)=0
$$

arising from the variation of the F-field. In the last two equations we denoted by $X^{\mu}$ the coordinates of the target space and by $Y^{a}$ the coordinates of the brane whose embedding is given by $X^{\mu}=X^{\mu}\left(Y^{a}\right)$. While the equations (35) are self-instructional the equations (34) require a bit of explanation. The main ingredient $\Omega_{a b}^{\mu}$ is a generalisation of the second fundamental form and takes into account the background fluxes. We may write

$$
\Omega_{a b}^{\mu}=\partial_{a} \partial_{b} X^{\mu}+\Gamma_{\nu \rho}^{\mu} \partial_{a} X^{\nu} \partial_{b} X^{\rho}-\hat{\Gamma}_{a b}^{c} \partial_{c} X^{\mu}
$$

The connections which enter the definition of this object are a combination of the Levi-Civita connection and the three-form flux,

$$
\Gamma=\Gamma(g)-\frac{1}{2} H \quad \Gamma(g)_{\lambda \mu \nu}=\frac{1}{2}\left(\partial_{\mu} g_{\lambda \nu}+\partial_{\nu} g_{\lambda \mu}-\partial_{\lambda} g_{\mu \nu}\right) .
$$

Hatted objects refer to induced quantities. In order to calculate $\hat{\Gamma}$ we use the previous formulas and plug in the induced H-field $\hat{H}$ and the Levi-Civita connection for the induced metric $\hat{g}$.

\subsubsection{Calculations for the branes in $S U(2)_{k_{1}} \times S U(2)_{k_{2}}$}

In order to check that the generalised permutation branes satisfy the equations of motion (34) and (35) a detailed knowledge of the induced metric, the induced H-field and the boundary two-form is required. The first two quantities are derived from the background fields on $S U(2)_{k_{1}} \times S U(2)_{k_{2}}$ which we will describe now. A particularly convenient parametrisation of $S U(2)$ is

$$
g=\left(\begin{array}{cc}
\cos \psi+i \cos \theta \sin \psi & \sin \psi \sin \theta e^{i \phi} \\
-\sin \psi \sin \theta e^{-i \phi} & \cos \psi-i \cos \theta \sin \psi
\end{array}\right) .
$$

In these coordinates the metric takes the form

$$
d s^{2}=k_{1}\left[d \psi_{1}^{2}+\sin ^{2} \psi_{1}\left(d \theta_{1}^{2}+\sin ^{2} \theta_{1} d \phi_{1}^{2}\right)\right]+k_{2}\left[d \psi_{2}^{2}+\sin ^{2} \psi_{2}\left(d \theta_{2}^{2}+\sin ^{2} \theta_{2} d \phi_{2}^{2}\right)\right] .
$$

A straightforward calculation yields the H-field

$$
H=2 k_{1} \sin ^{2} \psi_{1} \sin \theta_{1} d \psi_{1} \wedge d \theta_{1} \wedge d \phi_{1}+2 k_{2} \sin ^{2} \psi_{2} \sin \theta_{2} d \psi_{2} \wedge d \theta_{2} \wedge d \phi_{2} .
$$

Locally this can be integrated and gives rise to a B-field of the form

$$
B=k_{1}\left(\psi_{1}-\frac{1}{2} \sin \left(2 \psi_{1}\right)\right) \sin \theta_{1} d \theta_{1} \wedge d \phi_{1}+k_{2}\left(\psi_{2}-\frac{1}{2} \sin \left(2 \psi_{2}\right)\right) \sin \theta_{2} d \theta_{2} \wedge d \phi_{2} .
$$




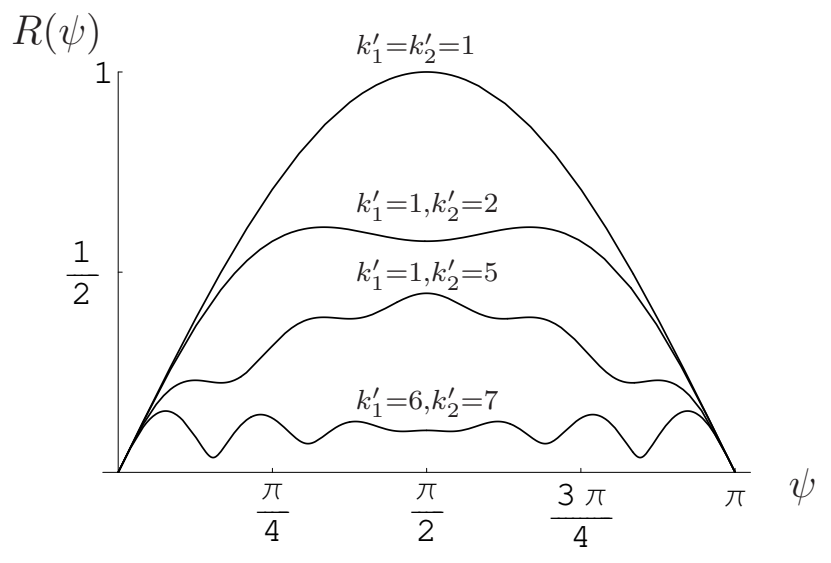

Figure 5: An illustration of the geometry of the three-dimensional brane for different values of $k_{1}^{\prime}$ and $k_{2}^{\prime}$ as an $S^{2}$-fibration over the interval $[0, \pi]$. The function $R(\psi)$ measures the radius of the two-sphere sitting over $\psi \in[0, \pi], d \hat{s}^{2} \propto d \psi^{2}+R(\psi)^{2}\left(d \theta^{2}+\sin ^{2} \theta d \phi^{2}\right)$.

This expression is valid on the whole space as long as $\psi_{i} \neq \pi$.

The crucial feature of the parametrisation (38) is that arbitrary powers of $g$ are under explicit control. Namely, the group element $g^{n}, n \in \mathbb{Z}$, has the same coordinates $\theta$ and $\phi$ as $g$ itself. Just the angle $\psi$ has to be replaced by $n \psi$. In the case of the lowest dimensional generalised permutation brane this property makes it particularly simple to determine the induced metric and the induced H-field. Let indeed $\left(g_{1}, g_{2}\right)=\left(g^{k_{2}^{\prime}}, g^{-k_{1}^{\prime}}\right)$ be an element of the brane (11), parametrised by angles $\psi_{i}, \theta_{i}, \phi_{i}$ and $\psi, \theta, \phi$, respectively. According to our previous statement the coordinates are related by $\psi_{1}=k_{2}^{\prime} \psi, \psi_{2}=-k_{1}^{\prime} \psi, \theta_{1}=\theta_{2}=\theta$ and $\phi_{1}=\phi_{2}=\phi$. The induced metric thus reads

$$
d \hat{s}^{2}=\frac{k_{1} k_{2}}{k}\left(k_{1}^{\prime}+k_{2}^{\prime}\right) d \psi^{2}+\left[k_{1} \sin ^{2} k_{2}^{\prime} \psi+k_{2} \sin ^{2} k_{1}^{\prime} \psi\right]\left(d \theta^{2}+\sin ^{2} \theta d \phi^{2}\right) .
$$

This geometry corresponds to a deformed sphere (see figure 5 for an illustration). The induced $\mathrm{H}$-field can easily be determined to be

$$
\hat{H}=\frac{k_{1} k_{2}}{k}\left[\cos \left(2 k_{1}^{\prime} \psi\right)-\cos \left(2 k_{2}^{\prime} \psi\right)\right] \sin \theta d \psi \wedge d \theta \wedge d \phi
$$

An explicit integration over the brane world volume shows that the total flux is zero. For the special choice $k_{1}=k_{2}$ the metric (42) reduces to that of a sphere. Note that at the same time the induced H-field vanishes.

Last but not least we have to derive the value of the boundary two-form. Our general formula (15) looks rather cumbersome. Fortunately it can also be determined more directly by integrating the relatively simple expression (43) for the induced H-field in our adapted coordinates. Following this route we easily find

$$
\omega_{C}=\frac{1}{2}\left[k_{2} \sin \left(2 k_{1}^{\prime} \psi\right)-k_{1} \sin \left(2 k_{2}^{\prime} \psi\right)\right] \sin \theta d \theta \wedge d \phi=\hat{B} .
$$


As indicated this coincides with the induced B-field meaning that there is no additional F-field on the brane. The sum of the induced metric and the boundary two-form which enters the DBI action is given by

$$
\hat{g}+\omega_{C}=\left(\begin{array}{ccc}
\frac{k_{1} k_{2}}{k}\left(k_{1}^{\prime}+k_{2}^{\prime}\right) & 0 & 0 \\
0 & p(\psi) & q(\psi) \sin \theta \\
0 & -q(\psi) \sin \theta & p(\psi) \sin ^{2} \theta
\end{array}\right)
$$

where

$$
p(\psi)=k_{1} \sin ^{2} k_{2}^{\prime} \psi+k_{2} \sin ^{2} k_{1}^{\prime} \psi \quad, \quad q(\psi)=\frac{1}{2}\left[k_{2} \sin \left(2 k_{1}^{\prime} \psi\right)-k_{1} \sin \left(2 k_{2}^{\prime} \psi\right)\right] .
$$

Now we can check the F-field equation of motion (35). The matrix that enters in this equation is

$$
\sqrt{\operatorname{det}\left(\hat{g}+\omega_{C}\right)}\left[\left(\hat{g}+\omega_{C}\right)^{-1}\right]_{\text {antisym }}^{a b}=\sqrt{\frac{k_{1} k_{2}\left(k_{1}^{\prime}+k_{2}^{\prime}\right)}{k\left(p(\psi)^{2}+q(\psi)^{2}\right)}} q(\psi)\left(\begin{array}{ccc}
0 & 0 & 0 \\
0 & 0 & -1 \\
0 & 1 & 0
\end{array}\right) .
$$

As this expression only depends on $\psi$, but all $\psi$-components of the matrix are zero, the equation (35) is satisfied.

To check the other DBI equation of motion (34) for our brane we have to combine all the given data into the generalised connections. Since the computations are straightforward but not particularly enlightening, we decided to collect the main intermediate steps in appendix B. Here we just state the final outcome that the equations (34) are indeed satisfied. These results imply that the submanifold (11) describes a brane in the geometric regime of string theory, i.e. a conformal boundary condition of the underlying world sheet theory.

So far we just discussed the degenerate case of the lowest dimensional brane. The other branes are not 3-dimensional but 5-dimensional submanifolds of $S U(2) \times S U(2)$. This is a major technical complication because in order to determine the induced metric and the other quantities which enter the DBI action (33) we have to be able to evaluate both, the powers

$$
\left(h_{1} f h_{2}^{-1}\right)^{k_{2}^{\prime}} \quad \text { and } \quad\left(h_{2} f h_{1}^{-1}\right)^{k_{1}^{\prime}},
$$

at the same time in a closed form. Since we have not been able to achieve this goal up to now, we restricted ourselves to a numerical check for a choice of low levels and a random selection of points on the group manifold. This numerical analysis gave a further confirmation for our claim that not only (11) but also the higher-dimensional geometries (10) describe true string theory branes.

\subsection{Brane tensions}

The brane tension is related to the g-factor in boundary conformal field theory [34, 35]. On the other hand we can determine this tension of a $p$-dimensional brane in the DBI formalism 
by evaluating the integral

$$
E=(2 \pi)^{-p} \int \sqrt{\operatorname{det}\left(\hat{g}+\omega_{C}\right)} .
$$

The normalisation corresponds to assigning the value $E=1$ to the D0-brane. Via their correspondence to g-factors the DBI energies can provide valuable hints for a CFT description of generalised permutation branes.

Let us start with the smallest, three-dimensional brane. Inserting the expression (45) for $g+\omega_{C}$ into the tension (48), we find

$$
\begin{aligned}
\mathcal{E}^{k_{1}, k_{2}}= & \frac{k^{3 / 2}}{2 \pi^{2}} \sqrt{k_{1}^{\prime} k_{2}^{\prime}\left(k_{1}^{\prime}+k_{2}^{\prime}\right)} \\
& \times \int_{0}^{\pi} d \psi \sqrt{\left(k_{1}^{\prime}+k_{2}^{\prime}\right)\left[k_{1}^{\prime} \sin ^{2}\left(k_{2}^{\prime} \psi\right)+k_{2}^{\prime} \sin ^{2}\left(k_{1}^{\prime} \psi\right)\right]-k_{1}^{\prime} k_{2}^{\prime} \sin ^{2}\left(k_{1}^{\prime}+k_{2}^{\prime}\right) \psi} .
\end{aligned}
$$

For equal levels this is easily evaluated to be

$$
\mathcal{E}^{k, k}=\frac{k^{3 / 2}}{\sqrt{2} \pi}
$$

For $k_{1}^{\prime}=1$ and $k_{2}^{\prime}=2$, the tension is given by an elliptic integral,

$$
\mathcal{E}^{k, 2 k}=\frac{k^{3 / 2}}{\pi^{2}} \sqrt{6}\left(E\left(\frac{1}{4}\right)+E\left(\frac{8}{9}\right)\right),
$$

where $E$ is the complete elliptic integral of the second kind.

The computation of the tension of the higher dimensional branes is more involved. Numerical studies indicate that the energies are given by

$$
E_{n}^{k_{1}, k_{2}}=\frac{\kappa}{\pi} \sin \left(\frac{\pi n}{\kappa}\right) \cdot \mathcal{E}^{k_{1}, k_{2}}
$$

for the brane with $\xi_{n}=\pi n / \kappa$. The energies of all generalised permutation branes may thus be expressed in terms of that of the simplest one.

It is interesting to note that the energy dependence on $n$ is simply given by a sine-factor which is proportional to the modular S-matrix of $\widehat{s u}(2)$ at level $\kappa-2$. This result can be translated into a prediction for the g-factors that we should expect in a CFT analysis. The g-factor is directly proportional to the tension; by using the correct normalisation (which we can get from a comparison with the untwisted Cardy branes), we obtain

$$
g_{n}^{k_{1}, k_{2}}=\left(k_{1}^{\prime} k_{2}^{\prime}\right)^{-1 / 4} \frac{\sin \frac{\pi n}{\kappa}}{\sqrt{\sin \frac{\pi}{k_{1}} \sin \frac{\pi}{k_{2}}}} \frac{\sqrt{2} \pi}{k^{3 / 2}} \mathcal{E}^{k_{1}, k_{2}} .
$$

This prediction, however, has to be taken with a pinch of salt. The DBI approach neglects higher order curvature corrections and can be trusted only for large $k$. In the supersymmetric 
model, one might hope that the DBI result predicts the exact CFT data as it is the case for the maximally symmetric branes. Indeed, for equal levels equation (53) reproduces precisely the known g-factors of ordinary permutation branes,

$$
g_{n}^{k, k}=\frac{S_{n-10}}{S_{00}}
$$

where $S$ is the modular S-matrix of $\widehat{s u}(2)_{k-2}$.

\subsection{Brane spectrum}

The semi-classical description of generalised permutation branes in $S U(2) \times S U(2)$ that we developed in the last few sections may be used to extract valuable information about the low-energy excitations of open strings which correspond to particle wave functions. Since our branes possess a residual $S U(2)$ symmetry we expect the spectrum to be organised in representations of $S U(2)$ and indeed this is what we will find.

\subsubsection{Derivation of the Laplacian}

In the presence of non-trivial fluxes the open strings see a deformed geometry. In order to determine the Laplacian on the brane we therefore have to use the open string metric (see e.g. [36])

$$
G=\hat{g}-\omega_{C} \hat{g}^{-1} \omega_{C}
$$

which does not only depend on the induced metric $\hat{g}$ on the brane as given in (42), but also on the boundary two-form $\omega_{C}$ which has been specified in (44). Plugging in the explicit values for the three-dimensional brane we find

$$
G=\left(\begin{array}{ccc}
c & 0 & 0 \\
0 & a(\psi) & 0 \\
0 & 0 & a(\psi) \sin ^{2} \theta
\end{array}\right)
$$

where the function $a(\psi)$ and the constant $c$ are given by

$$
\begin{aligned}
c & =k_{1}^{\prime} k_{2}^{\prime}\left(k_{1}+k_{2}\right) \\
a(\psi) & =\frac{4\left[k_{1} \sin ^{2}\left(k_{2}^{\prime} \psi\right)+k_{2} \sin ^{2}\left(k_{1}^{\prime} \psi\right)\right]^{2}+\left[k_{1} \sin ^{2}\left(2 k_{2}^{\prime} \psi\right)-k_{2} \sin ^{2}\left(2 k_{1}^{\prime} \psi\right)\right]^{2}}{4\left[k_{1} \sin ^{2}\left(k_{2}^{\prime} \psi\right)+k_{2} \sin ^{2}\left(k_{1}^{\prime} \psi\right)\right]} .
\end{aligned}
$$

The metric (56) clearly exhibits the spherical symmetry we expected from the start. In order to determine the spectrum we have to identify the normalisable eigenfunctions of the Laplacian

$$
\Delta f=-\frac{1}{\sqrt{G}} \partial_{a}\left[\sqrt{G} G^{a b} \partial_{b} f\right]=-\frac{1}{c}\left[\frac{a^{\prime}(\psi)}{a(\psi)} \partial_{\psi}+\partial_{\psi}^{2}\right] f+\frac{1}{a(\psi)} \Delta_{S^{2}} f
$$

Note that the dependence on $\theta$ and $\phi$ could be completely absorbed in the Laplacian $\Delta_{S^{2}}$ on a sphere. 
From the general structure of the previous expression we see that we can use the separation ansatz

$$
f_{l m}(\psi, \theta, \phi)=g_{l m}(\psi) Y_{l m}(\theta, \phi)
$$

which involves the spherical harmonics $Y_{l m}(\theta, \phi)$. Using the eigenvalue equation of the latter with respect to $\Delta_{S^{2}}$ we arrive at the ordinary differential equation

$$
a^{\prime}(\psi) g_{l m}^{\prime}+a(\psi) g_{l m}^{\prime \prime}-[l(l+1) c-\lambda c a(\psi)] g_{l m}=0
$$

which determines the spectrum of eigenvalues $\lambda$ on our brane. Since the function $a(\psi)$ is rather complicated it seems hopeless to solve this equation in full generality in terms of known functions. As we will see below the solution may be expressed in terms of associated Legendre functions in the case of equal levels. For the remaining cases, however, we restrict ourselves to a numerical analysis and to an estimate in the regime of large eigenvalues.

\subsubsection{The case of equal levels}

Let us now specialise to the case $k_{1}=k_{2}=k$. For these parameters eq. (56) reduces to

$$
G=\left(\begin{array}{ccc}
2 k & 0 & 0 \\
0 & 2 k \sin ^{2}(\psi) & 0 \\
0 & 0 & 2 k \sin ^{2}(\psi) \sin ^{2} \theta
\end{array}\right)
$$

This is simply the metric for an ordinary 3 -sphere at radius $\sqrt{2 k}$. Since $S^{3} \sim S U(2)$ we know that in this case the Laplacian is proportional to the quadratic Casimir operator and its spectrum of eigenfunctions can be deduced from the Peter-Weyl theorem. More precisely, the algebra of functions may be decomposed into irreducible representations $\mathcal{H}_{j}$ of $S U(2)$ according to

$$
\mathcal{F}\left(S^{3}\right)=\bigoplus_{j=0}^{\infty}(2 j+1) \mathcal{H}_{j}
$$

The semi-classical spectrum should be proportional to the quadratic Casimirs $j(j+1)$ where each eigenvalue has a degeneracy of $(2 j+1)^{2}$. As one may easily check, this prediction coincides with the CFT calculation for the 3-dimensional permutation brane in $S U(2)_{k} \times$ $S U(2)_{k}$ in the limit of large level $k$ (see e.g. [17, 37]).

In order to get some intuition how the solutions of eq. (61) look like, we would like to construct them explicitly and confirm the previous predictions. Using the ansatz

$$
g(\psi)=(\sin \psi)^{-\frac{1}{2}} h(\cos (\psi))
$$

and substituting $z=\cos (\psi)$, the original differential equation assumes the form

$$
\left(1-z^{2}\right) h^{\prime \prime}-2 z h^{\prime}+\left\{\nu(\nu+1)-\frac{\mu^{2}}{1-z^{2}}\right\} h=0
$$


of Legendre's differential equation with parameters given by

$$
\mu=-\left(l+\frac{1}{2}\right) \quad \nu=-\frac{1}{2}+\sqrt{1+2 k \lambda} .
$$

The solutions are associated Legendre functions $P_{\nu}^{\mu}(z)$ and the requirement of regularity of our solutions at $z= \pm 1$ leads to the constraint

$$
\mu+\nu=n \in \mathbb{N}_{0}
$$

Solving for the eigenvalue $\lambda$ we obtain

$$
\lambda=\frac{(n+l)(n+l+2)}{2 k}=\frac{2 j(j+1)}{k} .
$$

In the last step we identified $n+l$ with twice the $\operatorname{spin} j$. Let us finally determine the number of solutions with given eigenvalue $\lambda$. For fixed $l$ we have a degeneracy $2 l+1$ coming from the spherical harmonics. Altogether we thus have a multiplicity of

$$
\sum_{l=0}^{2 j}(2 l+1)=(2 j+1)^{2} .
$$

This precisely confirms our expectations.

\subsubsection{The general case and solutions for large eigenvalues $\lambda$}

For a detailed discussion of the differential equation (61) the ansatz

$$
g(\psi)=u(\psi) / \sqrt{a(\psi)}
$$

turns out to be very useful since it eliminates the first order term. The resulting equation for $u(\psi)$ is given by

$$
u^{\prime \prime}-f(\psi) u=0 \quad \text { with } \quad f(\psi)=\frac{2 a^{\prime \prime} a-a^{2}}{4 a^{2}}+l(l+1) \frac{c}{a}-\lambda c .
$$

From the definition (57) we infer that $a(\psi)$ is periodic with period $\pi$. Note also that the function $f(\psi)$ may be rewritten as a rational function in the variable $z=\cos (\psi)$, but the powers involved in the numerator and in the denominator will depend in a non-trivial way on the levels $k_{1}$ and $k_{2}$ and can be rather large. In fact the maximal power is not bounded when considered as a function of $k_{1}$ and $k_{2}$.

As a consequence we will not try to solve equation (71) in closed form in full generality. In a case by case study one might hope to find all the solutions explicitly for certain values of the levels. Here, however, we will focus our attention to the case of large eigenvalues $\lambda$. Under this assumption $\lambda$ will dominate the function $f(\psi)$ except when the latter becomes 
singular which happens precisely at the boundary $\psi=0, \pi$. Close to $\psi=0$ the function $a(\psi)$ possesses an expansion

$$
a(\psi)=\gamma^{-1} \psi^{2}+O\left(\psi^{4}\right) \quad \text { with } \quad \gamma^{-1}=\frac{k_{1}^{\prime} k_{2}^{\prime}\left(5 k_{1}^{2}-6 k_{1} k_{2}+5 k_{2}^{2}\right)}{k_{1}+k_{2}} .
$$

After calculating the derivatives one recognises that the first term in $f(\psi)$ is subleading at $\psi=0$. This observation motivates us to consider the differential equation

$$
u^{\prime \prime}-f_{s}(\psi) u=0 \quad \text { with } \quad f_{s}(\psi)=\frac{l(l+1) \gamma c}{\sin ^{2}(\psi)}-\lambda c
$$

instead of the original one. We replaced $f(\psi)$ by its singular part $f_{s}(\psi)$ which differs from $f(\psi)$ only by a regular function independent of $\lambda$. Dropping this regular part will change the eigenvalue $\lambda$ only by an amount which does not grow with $\lambda$. The new differential equation can be solved similarly to the case at equal levels by transforming it into Legendre's differential equation (65). Here the parameters are

$$
\mu=-\sqrt{l(l+1) \gamma c+\frac{1}{4}} \quad \nu=-\frac{1}{2}+\sqrt{\lambda c} .
$$

With the quantisation condition (67) we find for the eigenvalues $\lambda$ of the reduced equation (73)

$$
\lambda_{n}^{\text {approx }} c=\left(n+\frac{1}{2}+\sqrt{l(l+1) \gamma c+\frac{1}{4}}\right)^{2} .
$$

The true eigenvalues $\lambda_{n}$ differ from these by a shift which becomes independent of $n$,

$$
\lambda_{n} c=d\left(k_{1}^{\prime}, k_{2}^{\prime}, l\right)+\left(n+\frac{1}{2}+\sqrt{l(l+1) \gamma c+\frac{1}{4}}\right)^{2}+(\text { terms vanishing for } n \rightarrow \infty) .
$$

Comparing with the numerical results which are discussed below, this result describes the spectrum very well for large eigenvalues $\lambda$.

\subsubsection{Numerical analysis}

If the levels $k_{1}$ and $k_{2}$ are different, we could just identify the asymptotic form of the spectrum analytically. Here, we would like to determine the energy of the low lying excitations accurately by numerical methods. This provides us with an expectation for the lowest conformal weights of boundary fields in a CFT formulation. Furthermore, we can check whether there are degeneracies of eigenvalues which would signal an enhanced symmetry.

In the following we compute the spectrum numerically for the simplest non-trivial case of $k_{1}=1$ and $k_{2}=2$. As we mentioned before the differential equation (171) is periodic with period $\pi$ and invariant under $\psi \mapsto \pi-\psi$. The solutions thus split into two classes, those 


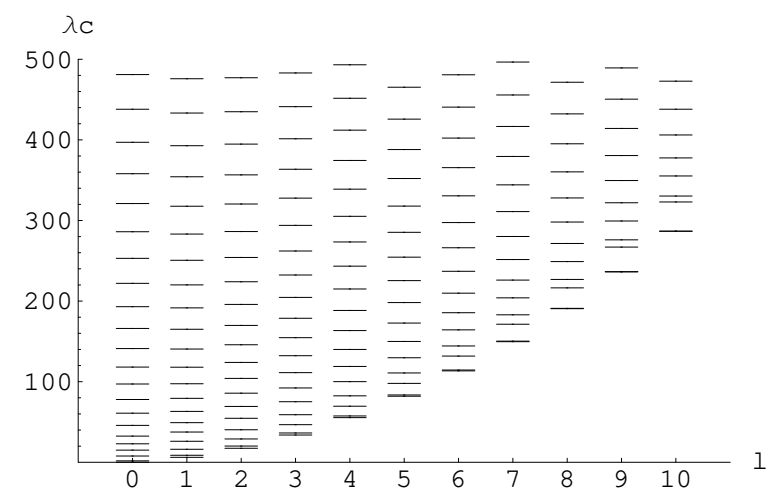

Figure 6: The spectrum of the 3-dimensional generalised permutation brane on $S U(2)_{1} \times$ $S U(2)_{2}$.

\begin{tabular}{|c|cccccccccc|}
\hline Quantum number $l$ & 0 & 0 & 1 & 0 & 1 & 0 & 1 & 2 & 2 & 0 \\
\hline Parity (even/odd) & $\mathrm{e}$ & $\mathrm{o}$ & $\mathrm{e}$ & $\mathrm{e}$ & $\mathrm{o}$ & $\mathrm{o}$ & $\mathrm{e}$ & $\mathrm{e}$ & $\mathrm{o}$ & $\mathrm{e}$ \\
\hline Energy $c \lambda$ & 0 & 1.78 & 6.14 & 7.75 & 8.70 & 15.07 & 16.14 & 17.38 & 20.14 & 22.92 \\
\hline Multiplicity & 1 & 1 & 3 & 1 & 3 & 1 & 3 & 5 & 5 & 1 \\
\hline
\end{tabular}

Table 1: Low lying excitations of the 3-dimensional generalised permutation brane on $S U(2)_{1} \times S U(2)_{2}$.

which are even and those which are odd under $\psi \mapsto \pi-\psi$. We determined numerically for which values of $\lambda$ the function with boundary values

$$
\text { i) } u(\pi / 2)=1, u^{\prime}(\pi / 2)=0 \quad \text { ii) } u(\pi / 2)=0, u^{\prime}(\pi / 2)=1
$$

can be extended to the boundary $\psi=\pi$ such that $u(\pi)=0$. For values of $\lambda$ which are not part of the spectrum the solution is singular and $u(\psi)$ will blow up. For $\lambda$ sufficiently close to an eigenvalue, the solution, however, will be sufficiently well-behaved close to $\psi=\pi$. Our findings are summarised in table 1 and plotted in figure 6. Our investigation shows that the large degeneracy of the eigenvalues that exists for equal levels is partially lifted. In the generic case only the $S U(2)$-degeneracy associated with the spherical harmonics survives.

\section{Generalisation to higher rank groups}

In the last section of this paper we would like to indicate how the previous ideas may be generalised to the product $G \times G$ of simple groups of higher rank. The presentation will closely follow the exposition in appendix A of [38. We restrict ourselves to the case of the lowest dimensional generalised permutation brane. 


\subsection{A useful parametrisation of simple groups}

Let $G$ be a compact simple simply-connected Lie group. As we saw for the simplest generalised permutation brane in $S U(2) \times S U(2)$, it is absolutely crucial to find a coordinate system in which an arbitrary power of a group element $g \in G$ can easily be determined if we want to perform explicit calculations. We follow [38] and use the parametrisation

$$
g=h^{-1}(\theta) t(\chi) h(\theta),
$$

where $h \in G$ and $t$ is an element of the Cartan torus $T$. There is some obvious redundancy under the replacement $h \mapsto f h$ with $f \in T$, such that $h$ in fact takes values in the coset $G / T$. Note that powers of $g$ are easily determined to be $g^{n}=h^{-1} t^{n} h$. In this sense the parametrisation (78) mimics the behaviour of the coordinates which we used on $S U(2) \times$ $S U(2)$ in the previous section. Let us emphasise that in contrast to [38] we are not assuming $t$ to be constant.

Our aim is to express the metric and the Wess-Zumino form in coordinates related to the decomposition (78) above. Let us introduce a Cartan-Weyl basis of $\mathfrak{g}$ consisting of Cartan elements $H_{i}$ and root generators $E^{\alpha}$. They satisfy the commutation relations

$$
\left[H_{i}, E^{\alpha}\right]=\alpha^{i} E^{\alpha} \quad\left[E^{\alpha}, E^{-\alpha}\right]=\alpha^{i} H_{i}
$$

and others which will not be important in the sequel but can be found in any text book or in [38. The operators are assumed to be orthonormal,

$$
\operatorname{tr}\left(H_{i} H_{j}\right)=\delta_{i j} \quad \operatorname{tr}\left(E^{\alpha} E^{\beta}\right)=\delta_{\alpha,-\beta},
$$

where ' $\operatorname{tr}$ ' denotes a suitably normalised trace. We will assume $t$ to be of the form $t=e^{i(\chi, H)}$.

For the purpose of explicit calculations it is convenient to introduce the one-forms

$$
t^{-1} d t=d t t^{-1}=i(d \chi, H) \quad \text { and } \quad d h h^{-1}=\sum_{\alpha>0}\left[\theta^{\alpha} E^{\alpha}-\bar{\theta}^{\alpha} E^{-\alpha}\right]+i(\zeta, H)
$$

For group elements $g$ close to the identity we will use $\chi$ and the complex numbers $\theta^{\alpha}$ as coordinates. The coordinate $\zeta$ is an auxiliary variable which will drop out of every physical expression in accordance with the gauge symmetry present in (78). In terms of the coordinates just introduced the (normalised) metric $d s^{2}=-\frac{k}{2} \operatorname{tr}\left[g^{-1} d g \otimes g^{-1} d g\right]$ admits the simple form

$$
d s^{2}=2 k \sum_{\alpha>0} \sin ^{2} \frac{1}{2}(\chi, \alpha)\left[\theta^{\alpha} \otimes \bar{\theta}^{\alpha}+\bar{\theta}^{\alpha} \otimes \theta^{\alpha}\right]+\frac{k}{2} \sum_{m} d \chi^{m} \otimes d \chi^{m} .
$$

If we had assumed $\chi$ to be constant, we would exactly recover the result of [38]. During the calculation we made use of the commutativity of elements in the Cartan subalgebra and we employed the formula

$$
\operatorname{Ad}_{t}\left(d h h^{-1}\right)=e^{i(\chi, \alpha)} \theta^{\alpha} E^{\alpha}-e^{-i(\chi, \alpha)} \bar{\theta}^{\alpha} E^{-\alpha}+i(\zeta, H)
$$


which follows from the commutation relations (79). This relation will be crucial in the following.

The same techniques can be used to calculate the Wess-Zumino form. With the help of the Polyakov-Wiegmann identity (12) we find

$$
\left.\omega^{\mathrm{WZ}}(g)\right|_{g=h^{-1} t h}=-\frac{k}{2} d \operatorname{tr}\left[\operatorname{Ad}_{t}\left(d h h^{-1}\right) \wedge d h h^{-1}+2 t^{-1} d t \wedge d h h^{-1}\right] .
$$

The term $\omega^{\mathrm{WZ}}(t)$ drops out because $t$ is an element of the Cartan torus. Using eq. (83) we may further simplify the previous expression. This manipulation yielde7

$$
\left.\omega^{\mathrm{WZ}}(g)\right|_{g=h^{-1} t h}=k d\left[i \sum_{\alpha>0} \sin (\chi, \alpha) \theta^{\alpha} \wedge \bar{\theta}^{\alpha}+(d \chi, \zeta)\right] .
$$

In the case of a constant $\chi$ one can immediately read off the boundary two-form for untwisted branes on the group manifold $G$. In this paper, however, we are interested in generalised permutation branes in the product $G \times G$ and thus there is still some work to do.

\subsection{Application to the product case}

Let us now consider the product group $G \times G$ at levels $k_{1}$ and $k_{2}$. If we choose real coordinates $\chi_{i}$ and complex coordinates $\theta_{i}^{\alpha}$ for the elements $g_{i}$ of the two individual factors as in the previous section, then the metric is given by

$$
d s^{2}=\sum_{j=1,2} k_{j}\left\{2 \sum_{\alpha>0} \sin ^{2} \frac{1}{2}\left(\chi_{j}, \alpha\right)\left[\theta_{j}^{\alpha} \otimes \bar{\theta}_{j}^{\alpha}+\bar{\theta}_{j}^{\alpha} \otimes \theta_{j}^{\alpha}\right]+\frac{1}{2} \sum_{m} d \chi_{j}^{m} \otimes d \chi_{j}^{m}\right\} .
$$

Since we have chosen our parametrisation such that $g^{n}=h^{-1} t^{n} h$, we can easily determine the induced metric on the generalised permutation brane (111). We just have to set $\chi_{1}=k_{2}^{\prime} \chi$ and $\chi_{2}=-k_{1}^{\prime} \chi$ while $\theta_{1}^{\alpha}=\theta_{2}^{\alpha}=\theta^{\alpha} 8$ The induced metric for our simplest branes is thus

$$
\begin{aligned}
d \hat{s}^{2}= & 2 \sum_{\alpha>0}\left[k_{1} \sin ^{2} \frac{k_{2}^{\prime}}{2}(\chi, \alpha)+k_{2} \sin ^{2} \frac{k_{1}^{\prime}}{2}(\chi, \alpha)\right]\left[\theta^{\alpha} \otimes \bar{\theta}^{\alpha}+\bar{\theta}^{\alpha} \otimes \theta^{\alpha}\right] \\
& +\frac{k_{1} k_{2}}{2 k}\left(k_{1}^{\prime}+k_{2}^{\prime}\right) \sum_{m} d \chi^{m} \otimes d \chi^{m} .
\end{aligned}
$$

The Wess-Zumino form may be calculated by adding up the contributions (85) for the two individual factors,

$$
\omega^{\mathrm{WZ}}\left(g_{1}, g_{2}\right)=\sum_{j=1,2} k_{j} d\left[i \sum_{\alpha>0} \sin \left(\chi_{j}, \alpha\right) \theta_{j}^{\alpha} \wedge \bar{\theta}_{j}^{\alpha}+\left(d \chi_{j}, \zeta_{j}\right)\right] .
$$

\footnotetext{
${ }^{7}$ It is not entirely surprising that this expression depends on the unphysical parameter $\zeta$. In fact, we could never expect the Wess-Zumino form to be exact. The occurrence of $\zeta$ is a remnant of this observation. The dependence on $\zeta$ should disappear if we expand the expression as a genuine three-form using the Maurer-Cartan equations.

${ }^{8} \mathrm{We}$ should also add $\zeta_{1}=\zeta_{2}=\zeta$ for completeness.
} 
If we restrict this expression to the brane, the terms with the unphysical parameters $\zeta_{i}$ cancel out and one can immediately read off the boundary two-form

$$
\omega_{C}=i\left[k_{1} \sin k_{2}^{\prime}(\chi, \alpha)-k_{2} \sin k_{1}^{\prime}(\chi, \alpha)\right] \theta^{\alpha} \wedge \bar{\theta}^{\alpha} .
$$

The relevant combination of metric and boundary two-form is thus given by

$$
\begin{aligned}
\hat{g}+\omega_{C}= & \frac{k_{1} k_{2}}{2 k}\left(k_{1}^{\prime}+k_{2}^{\prime}\right) \sum_{m} d \chi^{m} \otimes d \chi^{m} \\
& +i \sum_{\alpha>0}\left[k_{1} \sin k_{2}^{\prime}(\chi, \alpha)-k_{2} \sin k_{1}^{\prime}(\chi, \alpha)\right]\left(\theta^{\alpha} \otimes \bar{\theta}^{\alpha}-\bar{\theta}^{\alpha} \otimes \theta^{\alpha}\right) \\
& +2 \sum_{\alpha>0}\left[k_{1} \sin ^{2} \frac{k_{2}^{\prime}}{2}(\chi, \alpha)+k_{2} \sin ^{2} \frac{k_{1}^{\prime}}{2}(\chi, \alpha)\right]\left(\theta^{\alpha} \otimes \bar{\theta}^{\alpha}+\bar{\theta}^{\alpha} \otimes \theta^{\alpha}\right)
\end{aligned}
$$

This may also be written as

$$
\begin{aligned}
\hat{g}+\omega_{C}= & \frac{k_{1} k_{2}}{2 k}\left(k_{1}^{\prime}+k_{2}^{\prime}\right) \sum_{m} d \chi^{m} \otimes d \chi^{m} \\
& +\sum_{\alpha>0}\left[k_{1}\left(1-e^{-i k_{2}^{\prime}(\chi, \alpha)}\right)+k_{2}\left(1-e^{i k_{1}^{\prime}(\chi, \alpha)}\right)\right] \theta^{\alpha} \otimes \bar{\theta}^{\alpha} \\
& +\sum_{\alpha>0}\left[k_{1}\left(1-e^{i k_{2}^{\prime}(\chi, \alpha)}\right)+k_{2}\left(1-e^{-i k_{1}^{\prime}(\chi, \alpha)}\right)\right] \bar{\theta}^{\alpha} \otimes \theta^{\alpha} .
\end{aligned}
$$

The structure of this matrix is very simple,

$$
\hat{g}+\omega_{C}=\left(\begin{array}{ccc}
A & 0 & 0 \\
0 & 0 & B \\
0 & \bar{B} & 0
\end{array}\right)
$$

with diagonal matrices $A$ and $B$, and the determinant can easily be calculated,

$$
\begin{aligned}
\operatorname{det}\left(\hat{g}+\omega_{C}\right)=2^{\left|\Delta_{+}\right|}\left[\frac{k_{1} k_{2}}{k}\left(k_{1}^{\prime}+k_{2}^{\prime}\right)\right]^{r} & \prod_{\alpha>0}\left\{\left(k_{1}+k_{2}\right)^{2}+k_{1} k_{2}\left[\cos \left(k_{1}^{\prime}+k_{2}^{\prime}\right)(\chi, \alpha)-1\right]\right. \\
& \left.-\left(k_{1}+k_{2}\right)\left[k_{1} \cos k_{2}^{\prime}(\chi, \alpha)+k_{2} \cos k_{1}^{\prime}(\chi, \alpha)\right]\right\} .
\end{aligned}
$$

This expressions may be used to partially check the DBI equations of motion. We refrain from calculating the generalised second fundamental form (36) which is needed to verify the eqs. (34). The validity of the F-field equations (35), however, follows immediately from the fact that the only dependence of $\hat{g}+\omega_{C}$ is on $\chi$ and that the corresponding entries in its inverse cancel out due to the antisymmetrisation 9

\footnotetext{
${ }^{9}$ This argument is not entirely correct because one has to check that the one-forms $\theta$ provide good coordinates. A more careful analysis has by now been accomplished in [39].
} 


\section{$5 \quad$ Summary and outlook}

In this paper we presented evidence for the existence of new branes in the product $G \times G$ of Lie groups at different levels. They generalise the conventional permutation branes which exist for equal levels, but in contrast to the latter they are not maximally symmetric. For the string background $S U(2)_{k_{1}} \times S U(2)_{k_{2}}$ we could show explicitly that the proposed branes are solutions of Dirac-Born-Infeld theory. Moreover, their tensions revealed a surprising link to quantities connected with a single $S U(2)$ model at level $\kappa=\operatorname{lcm}\left(k_{1}, k_{2}\right)$. We also discussed the spectrum of excitations of the open string and found that the group theoretical degeneracy is partially lifted compared to the case of equal levels. Last but not least, the geometry of the branes suggests a very natural and complete explanation of the Ktheory charges for this particular product group. We hope that the semi-classical data and observations we compiled here will facilitate a treatment of these branes in the framework of exact CFT in the near future.

The basic construction we presented in this paper calls for generalisations in many different directions. First of all it is straightforward to combine the twist (8) with automorphisms which act on the single factors of the product group. The necessary modification of our formulas is obvious. As a consequence every pair of automorphisms of $G$ yields not only the usual factorising branes, but in addition the same number of new permutation branes. In the case of $S U(3) \times S U(3)$, these branes already account for all K-theory charges. For a general simple group $G$, the situation is less obvious. If we knew how to construct all branes in $G$ required by K-theory, then we would easily obtain all charge carrying factorising branes in the product group $G \times G$. They, however, could only account for half of the charges. It is thus likely that to every construction of factorising branes there is a corresponding "permuted" construction which contributes the remaining charges. Similar considerations can be applied to product groups consisting of more than two identical simple group factors. In that case the permutation group allows cycles of higher order which will lead to new classes of generalised permutation branes.

It is evident that our work just provides a glimpse onto phenomena in a rich but vastly unexplored landscape of models. In particular, product groups $G \times G$ in general cannot be part of a consistent string theory background - except for $S U(2) \times S U(2)$, Abelian groups and some low level examples - already for dimensional reasons. Instead what we are truly interested in are coset theories and products thereof. We are convinced that our generalised permutation branes have a direct analogue in coset spaces. In fact it is already known for a long time that a large class of cosets arises at the boundary of moduli space of currentcurrent deformations of WZNW models [40]. Such deformations can also be performed in the presence of a brane and it was shown using semi-classical methods [41] that the maximally symmetric branes in $S U(2)$ are deformed into the $A$ - and $B$-type branes of the parafermions $\mathrm{PF}=S U(2) / U(1)[14$.

We expect that a similar reasoning is applicable if one starts with one of our generalised permutation branes in $S U(2)_{k_{1}} \times S U(2)_{k_{2}}$ and deforms the background geometry. At the endpoint of the deformation one would be left with a presumably non-factorising brane in 
the product coset $\mathrm{PF}_{k_{1}} \times \mathrm{PF}_{k_{2}} 10$ At least we know that non-factorising permutation branes in the product of parafermions exist for $k_{1}=k_{2}$ and there is no reason to believe that this should be different if the levels are distinct. A natural proposal for the lowest dimensional generalised permutation brane in a coset $G_{k_{1}} / H \times G_{k_{2}} / H$ appears to be

$$
\mathcal{D}_{\tau}(e, e)=\left\{\left((g h)^{k_{2}^{\prime}},(h g)^{-k_{1}^{\prime}}\right) \mid g \in G, h \in H\right\} \subset G \times G
$$

with $k_{i}^{\prime}=k_{i} / \operatorname{gcd}\left(k_{1}, k_{2}\right)$. This set is indeed invariant under the adjoint action of $H \times H$ and defines therefore a candidate for a brane on the coset. Preliminary DBI calculations, however, seem to indicate that this geometry is not correct, calling for a more elaborate proposal.

An extension of our results to cosets is particularly desirable in view of recent developments in understanding branes in products of $N=2$ minimal models which, as a coset, are rather similar to the parafermions. Due to the presence of $N=2$ supersymmetry one can perform a topological twist and analyse the topological subsector of the original theory. This simplifies many of the calculations. In particular, the classification of B-branes can be reduced to the purely algebraic problem of the classification of matrix factorisations of the superpotential (this goes back to an unpublished idea of Kontsevich). In the corresponding investigations for products of minimal models a special class of branes has been discovered which just emerges if the levels involved have common divisors [21. Moreover, these branes provide an important contribution to the lattice of K-theory charges [42]. In this respect and also in the concrete expressions for the matrix factorisations they bear a close resemblance to the generalised permutation branes presented here.

The investigation of non-trivial branes in product CFTs has interesting applications. In string theory products of $N=2$ minimal models arise naturally in Gepner models. Also in statistical physics boundary conditions in products of CFTs play a distinguished role since they may be mapped to defect lines between the individual constituents using the folding trick [43]. Up to now, however, the classification of conformal defect lines is stuck at a rather preliminary stage, see e.g. [15] and references therein.

Summarising the last few paragraphs, there is by now an overwhelming evidence for the existence and importance of generalised permutation branes from several directions. What we still lack is an exact CFT prescription or even an idea what the precise infinite dimensional symmetry could be that is preserved by the branes. At the moment there is not much we can say about these issues but we would at least like to indicate where we see the best chances of making progress.

The most promising candidate for progress on the CFT side seem to be the aforementioned products of $N=2$ minimal models. For these theories one might expect to be able to combine the rather complementary information from the topological sector and the semi-classical regime into concrete guidelines for identifying the symmetry preserved and the construction of boundary states. On the other hand, the superconformal symmetry with its severe restrictions might also assist in mastering this task.

\footnotetext{
${ }^{10}$ Using the same idea, one could probably even have "permutation branes" in product CFTs such as $S U(2)_{k_{1}} \times \mathrm{PF}_{k_{2}}$ where one factor is a WZNW model while the other one is a coset.
} 
Another interesting idea is to consider generalised permutation branes in the product of ordinary minimal models. It is known that each minimal model $\mathcal{M}_{m}$ possesses a relevant integrable perturbation which connects it to the minimal model $\mathcal{M}_{m-1}$ in the infrared. In the simplest case of the tricritical Ising model $\mathcal{M}_{4}$, the flow to the Ising model $\mathcal{M}_{3}$ has been analysed in detail in [44, even in the presence of a boundary. One of the main results was an explicit map between initial and final boundary conditions along the flow. We hope that also the deformation of ordinary permutation branes in $\mathcal{M}_{4} \times \mathcal{M}_{4}$ can be analysed in the same spirit if we perturb the model towards $\mathcal{M}_{4} \times \mathcal{M}_{3}$. The major complication compared to the case of a single minimal model is that the final brane will probably not preserve the whole symmetry $\mathcal{M}_{4} \times \mathcal{M}_{3}$.

Let us finally comment on one of the big unsolved problems in CFT: the full classification of (super)conformal boundary conditions for a given background. Only partial results in this direction are available up to now [45, 46, 47, 15, 48. A detailed knowledge about the CFT construction of generalised permutation branes in product groups and cosets would certainly be a major step forward. In this paper it turned out to be extremely fruitful to accept the guidance of geometric and K-theoretic arguments and probably this will also be the case for other branes of particular physical significance. Eventually, new insights into the classification of branes or into the brane charges in certain backgrounds could be gained by combining the nested coset construction of branes [15, 16] with generalised permutations in common subfactors.

We hope to return to some of the open issues in future publications.

\section{Acknowledgements}

We are very grateful to Anton Alekseev for initial collaboration on this project. We also appreciate discussions with Costas Bachas, Volker Braun, Matthias Gaberdiel, Sylvain Ribault, Volker Schomerus and Gerard Watts. This work was partially supported by the EU Research Training Network grants "Euclid", contract number HPRN-CT-2002-00325, "Superstring Theory", contract number MRTN-CT-2004-512194, and "ForcesUniverse", contract number MRTN-CT-2004-005104. The work of SF is supported by the Max Planck Institute for Gravitational Physics and the Max Planck Society. The research of TQ is financed by a PPARC postdoctoral fellowship under reference PPA/P/S/2002/00370. He also receives partial support from the PPARC rolling grant PPA/G/O/2002/00475. This work was initiated during a stay of SF and TQ at the Section de Mathématiques, Université de Genève, which was enabled by the Swiss National Science Foundation.

\section{A K-theory for products of groups}

In this section we want to determine the twisted K-theory of $G \times G$. For this we employ the Künneth exact sequence (see e.g. [49]),

$$
0 \longrightarrow{ }^{\tau_{1}} K(G) \otimes^{\tau_{2}} K(G) \longrightarrow{ }^{\tau_{1}+\tau_{2}} K(G \times G) \longrightarrow \operatorname{Tor}\left({ }^{\tau_{1}} K(G),{ }^{\tau_{2}} K(G)\right) \longrightarrow 0
$$


Here, $\tau_{i}$ are elements of the third integral cohomology group of $G$; these are the WessZumino forms in the corresponding WZNW models. The sequence splits (unnaturally) so that we can determine ${ }^{\tau_{1}+\tau_{2}} K(G \times G)$ by computing the tensor product and the Tor-part.

The twisted K-theory of a simple, simply connected Lie group has been determined in [38, 9, 10, 11]. It is given by

$$
{ }^{\tau} K(G)=\left(\mathbb{Z}_{d}\right)^{2^{r-1}}
$$

where $r$ is the rank of $G$, and the order $d$ depends on $G$ and $\tau$. The tensor product of two of these K-groups is

$$
{ }^{\tau_{1}} K(G) \otimes{ }^{\tau_{2}} K(G) \cong\left(\mathbb{Z}_{\operatorname{gcd}\left(d_{1}, d_{2}\right)}\right)^{2^{2(r-1)}}
$$

Tor is the derived functor of the tensor product functor. To compute it we first have to find a resolution of ${ }^{\tau_{i}} K(G)$, i.e. a free chain complex with zeroth cohomology equal to ${ }^{\tau_{i}} K(G)$,

$$
0 \longrightarrow \mathbb{Z}^{2^{r-1}} \stackrel{\cdot d_{i}}{\longrightarrow} \underline{\mathbb{Z}}^{2^{r-1}} \longrightarrow 0
$$

The zeroth position is underlined. We take the tensor product of the chain complexes,

$$
0 \longrightarrow \mathbb{Z}^{2^{2(r-1)}} \stackrel{\left(\cdot d_{1}, d_{2}\right)}{\longrightarrow} \mathbb{Z}^{2^{2(r-1)}} \oplus \mathbb{Z}^{2^{2(r-1)}} \stackrel{\left(-d_{2}, d_{1}\right)}{\longrightarrow} \underline{\mathbb{Z}}^{2^{2(r-1)}} \longrightarrow 0
$$

The zeroth cohomology of this complex is

$$
\operatorname{Tor}\left({ }^{\tau_{1}} K(G),{ }^{\tau_{2}} K(G)\right) \cong\left(\mathbb{Z}_{\operatorname{gcd}\left(d_{1}, d_{2}\right)}\right) 2^{2^{2(r-1)}} .
$$

In total we find

$$
\tau_{1}+\tau_{2} K(G \times G) \cong\left(\mathbb{Z}_{\operatorname{gcd}\left(d_{1}, d_{2}\right)}\right)^{2^{2 r-1}} .
$$

Let us look at the example $G=S U(2)$. For the simple factors, the twisted K-theory is given by ${ }^{\tau_{i}} K(S U(2))=\mathbb{Z}_{k_{i}}$, where $k_{i}$ is the level of the corresponding WZNW model. The twisted K-theory of the product group is then

$$
\tau_{1}+\tau_{2} K(S U(2) \times S U(2)) \cong \mathbb{Z}_{\operatorname{gcd}\left(k_{1}, k_{2}\right)} \oplus \mathbb{Z}_{\operatorname{gcd}\left(k_{1}, k_{2}\right)},
$$

where one of the summands contributes to $K^{0}$ and the other to $K^{1}$.

\section{B Details of the Born-Infeld calculation}

In this appendix we collect some formulas which are needed to check the DBI equations of motion (34) in section 3.2.2. We will present explicit expressions for the generalised connections (37) based on the metrics (39) and (42) as well as on the H-fields (40) and (43). All expressions in this appendix are based on the parametrisation (38).

Since the embedding of the brane into the target space is expressed in terms of a linear relation, there is no contribution of the first term to the generalised second fundamental form (36). The derivatives in the second term just contribute constant factors. We write

$$
\Omega_{a b}^{\mu}=\Gamma_{\nu \rho}^{\mu} \partial_{a} X^{\nu} \partial_{b} X^{\rho}-\hat{\Gamma}_{a b}^{c} \partial_{c} X^{\mu}=\Omega_{a b}^{(1) \mu}+\Omega_{a b}^{(2) \mu}
$$


In order to determine the first contribution $\Omega_{a b}^{(1) \mu}$ we start with the Levi-Civita connection for the individual group factors for which we find

$$
\begin{array}{rlrl}
\Gamma(g)_{\psi_{i} \theta_{i} \theta_{i}} & =-k_{i} \sin \psi_{i} \cos \psi_{i} & \Gamma(g)_{\psi_{i} \phi_{i} \phi_{i}}=-k_{i} \sin \psi_{i} \cos \psi_{i} \sin ^{2} \theta_{i} \\
\Gamma(g)_{\theta_{i} \phi_{i} \phi_{i}}=-k_{i} \sin ^{2} \psi_{i} \sin \theta_{i} \cos \theta_{i} & \Gamma(g)_{\theta_{i} \psi_{i} \theta_{i}}=k_{i} \sin \psi_{i} \cos \psi_{i} \\
\Gamma(g)_{\phi_{i} \psi_{i} \phi_{i}}=k_{i} \sin \psi_{i} \cos \psi_{i} \sin ^{2} \theta_{i} & \Gamma(g)_{\phi_{i} \theta_{i} \phi_{i}}=k_{i} \sin ^{2} \psi_{i} \sin \theta_{i} \cos \theta_{i} .
\end{array}
$$

The remaining non-vanishing entries follow from the symmetry of the connection in the last two indices. By subtracting the individual $\mathrm{H}$-fields and raising the first index we obtain the generalised connection

$$
\begin{aligned}
\Gamma^{\psi_{i}} & =\left(\begin{array}{ccc}
0 & 0 & 0 \\
0 & -\sin \psi_{i} \cos \psi_{i} & -\sin ^{2} \psi_{i} \sin \theta_{i} \\
0 & \sin ^{2} \psi_{i} \sin \theta_{i} & -\sin \psi_{i} \cos \psi_{i} \sin ^{2} \theta_{i}
\end{array}\right) \\
\Gamma^{\theta_{i}} & =\left(\begin{array}{ccc}
0 & \cot \psi_{i} & \sin \theta_{i} \\
\cot \psi_{i} & 0 & 0 \\
-\sin \theta_{i} & 0 & -\sin \theta_{i} \cos \theta_{i}
\end{array}\right), \quad \Gamma^{\phi_{i}}=\left(\begin{array}{ccc}
0 & -\csc \theta_{i} & \cot \psi_{i} \\
\csc \theta_{i} & 0 & \cot \theta_{i} \\
\cot \psi_{i} & \cot \theta_{i} & 0
\end{array}\right) .
\end{aligned}
$$

Taking the additional factors into account which come from the derivatives in (102) and restricting the coordinates to the brane we easily find

$$
\begin{aligned}
& \Omega^{(1) \psi_{1}}=\left(\begin{array}{ccc}
0 & 0 & 0 \\
0 & -\sin \left(k_{2}^{\prime} \psi\right) \cos \left(k_{2}^{\prime} \psi\right) & -\sin ^{2}\left(k_{2}^{\prime} \psi\right) \sin \theta \\
0 & \sin ^{2}\left(k_{2}^{\prime} \psi\right) \sin (\theta) & -\sin \left(k_{2}^{\prime} \psi\right) \cos \left(k_{2}^{\prime} \psi\right) \sin ^{2} \theta
\end{array}\right)+\left(k_{2}^{\prime} \rightarrow-k_{1}^{\prime}\right) \\
& \Omega^{(1) \theta_{1}}=\left(\begin{array}{ccc}
0 & k_{2}^{\prime} \cot \left(k_{2}^{\prime} \psi\right) & k_{2}^{\prime} \sin \theta \\
k_{2}^{\prime} \cot \left(k_{2}^{\prime} \psi\right) & 0 & 0 \\
-k_{2}^{\prime} \sin \theta & 0 & -\sin \theta \cos \theta
\end{array}\right)+\left(k_{2}^{\prime} \rightarrow-k_{1}^{\prime}\right) \\
& \Omega^{(1) \phi_{1}}=\left(\begin{array}{ccc}
0 & -k_{2}^{\prime} \csc \theta & k_{2}^{\prime} \cot \left(k_{2}^{\prime} \psi\right) \\
k_{2}^{\prime} \csc \theta & 0 & \cot \theta \\
k_{2}^{\prime} \cot \left(k_{2}^{\prime} \psi\right) & \cot \theta & 0
\end{array}\right)+\left(k_{2}^{\prime} \rightarrow-k_{1}^{\prime}\right) .
\end{aligned}
$$

Similar, though slightly more cumbersome, calculations have to be performed for the induced quantities. Let us first introduce the abbreviations

$$
\begin{array}{ll}
r(\psi)=k_{1} \sin ^{2}\left(k_{2}^{\prime} \psi\right)+k_{2} \sin ^{2}\left(k_{1}^{\prime} \psi\right) & s(\psi)=k_{2} \sin \left(k_{1}^{\prime} \psi\right) \cot \left(k_{2}^{\prime} \psi\right)-k_{1} \cos \left(k_{1}^{\prime} \psi\right) \\
t(\psi)=\sin \left(2 k_{1}^{\prime} \psi\right)+\sin \left(2 k_{2}^{\prime} \psi\right) & u(\psi)=\sin ^{2}\left(k_{2}^{\prime} \psi\right)-\sin ^{2}\left(k_{1}^{\prime} \psi\right),
\end{array}
$$

which allow a compact representation of our results. The matrices of the generalised induced 
connection where the first index has been raised read

$$
\begin{aligned}
\hat{\Gamma}^{\psi} & =-\frac{1}{2\left(k_{1}^{\prime}+k_{2}^{\prime}\right)}\left(\begin{array}{ccc}
0 & 0 & 0 \\
0 & t(\psi) & u(\psi) \sin \theta \\
0 & -u(\psi) \sin \theta & t(\psi) \sin ^{2} \theta
\end{array}\right) \\
\hat{\Gamma}^{\theta} & =\frac{1}{r(\psi)}\left(\begin{array}{ccc}
0 & \frac{k_{1} k_{2}}{2 k} t(\psi) & \frac{k_{1} k_{2}}{2 k} u(\psi) \sin \theta \\
\frac{k_{1} k_{2}}{2 k} t(\psi) & 0 & 0 \\
-\frac{k_{1} k_{2}}{2 k} u(\psi) \sin \theta & 0 & -r(\psi) \sin \theta \cos \theta
\end{array}\right) \\
\hat{\Gamma}^{\phi} & =\frac{1}{r(\psi) \sin \theta}\left(\begin{array}{ccc}
0 & -\frac{k_{1} k_{2}}{2 k} u(\psi) & \frac{k_{1} k_{2}}{2 k} t(\psi) \sin \theta \\
\frac{k_{1} k_{2}}{2 k} u(\psi) & 0 & r(\psi) \cos \theta \\
\frac{k_{1} k_{2}}{2 k} t(\psi) \sin \theta & r(\psi) \cos \theta & 0
\end{array}\right) .
\end{aligned}
$$

Finally we are prepared to state the result for the complete generalised second fundamental form

$$
\begin{aligned}
\Omega^{\psi_{1}} & =\frac{1}{2\left(k_{1}^{\prime}+k_{2}^{\prime}\right)}\left(\begin{array}{ccc}
0 & 0 & 0 \\
0 & k_{2}^{\prime} \sin \left(2 k_{1}^{\prime} \psi\right)-k_{1}^{\prime} \sin \left(2 k_{2}^{\prime} \psi\right) & -2 r(\psi) \sin \theta \\
0 & 2 r(\psi) \sin \theta & {\left[k_{2}^{\prime} \sin \left(2 k_{1}^{\prime} \psi\right)-k_{1}^{\prime} \sin \left(2 k_{2}^{\prime} \psi\right)\right] \sin ^{2} \theta}
\end{array}\right) \\
\Omega^{\theta_{1}} & =\frac{1}{r(\psi)}\left(\begin{array}{ccc}
k_{2}^{\prime} \sin \left(k_{1}^{\prime} \psi\right) s(\psi) & k_{2}^{\prime} \sin \left(k_{1}^{\prime} \psi\right) s(\psi) & k_{2}^{\prime}\left(k_{1}+k_{2}\right) \sin ^{2}\left(k_{1}^{\prime} \psi\right) \sin \theta \\
-k_{2}^{\prime}\left(k_{1}+k_{2}\right) \sin ^{2}\left(k_{1}^{\prime} \psi\right) \sin \theta & 0 & 0 \\
0 & 0 & 0
\end{array}\right) \\
\Omega^{\phi_{1}} & =\frac{1}{r(\psi) \sin \theta}\left(\begin{array}{ccc}
k_{2}^{\prime}\left(k_{1}+k_{2}\right) \sin ^{2}\left(k_{1}^{\prime} \psi\right) & -k_{2}^{\prime}\left(k_{1}+k_{2}\right) \sin ^{2}\left(k_{1}^{\prime} \psi\right) & k_{2}^{\prime} \sin \left(k_{1}^{\prime} \psi\right) s(\psi) \sin \theta \\
k_{2}^{\prime} \sin \left(k_{1}^{\prime} \psi\right) s(\psi) \sin \theta & 0 & 0
\end{array}\right) .
\end{aligned}
$$

The second set of matrices with superscripts $\psi_{2}, \theta_{2}, \phi_{2}$ can easily be obtained from the previous ones.

One finally has to plug all these expressions into the equations of motion (34). For the labels $\mu=\theta_{1}$ and $\mu=\phi_{1}$ they are easily seen to be identically satisfied just because of the matrix structure which renders the trace zero. Only for $\mu=\psi_{1}$ one has to perform a small calculation to verify the equations of motion.

\section{References}

[1] R. Minasian and G. Moore, K-theory and Ramond-Ramond charge, JHEP 11 (1997) 002 hep-th/9710230.

[2] E. Witten, D-branes and K-theory, JHEP 12 (1998) 019 hep-th/9810188.

[3] P. Bouwknegt and V. Mathai, D-branes, B-fields and twisted K-theory, JHEP 03 (2000) 007 hep-th/0002023. 
[4] A. Yu. Alekseev and V. Schomerus, RR charges of D2-branes in the WZW model, hep-th/0007096.

[5] S. Fredenhagen and V. Schomerus, Branes on group manifolds, gluon condensates, and twisted K-theory, JHEP 04 (2001) 007 hep-th/0012164.

[6] M. R. Gaberdiel and T. Gannon, The charges of a twisted brane, JHEP 01 (2004) 018 hep-th/0311242.

[7] J. L. Cardy, Boundary conditions, fusion rules and the Verlinde formula, Nucl. Phys. B324 (1989) 581.

[8] L. Birke, J. Fuchs and C. Schweigert, Symmetry breaking boundary conditions and WZW orbifolds, Adv. Theor. Math. Phys. 3 (1999) 671-726 hep-th/9905038.

[9] V. Braun, Twisted K-theory of Lie groups, JHEP 03 (2004) 029 hep-th/0305178.

[10] D. S. Freed, M. J. Hopkins and C. Teleman, Twisted K-theory and loop group representations, math.at/0312155.

[11] C. Douglas, On the twisted K-homology of simple Lie groups, math.at/0402082.

[12] M. R. Gaberdiel, T. Gannon and D. Roggenkamp, The D-branes of SU(n), JHEP 07 (2004) 015 hep-th/0403271.

[13] M. R. Gaberdiel, T. Gannon and D. Roggenkamp, The coset D-branes of SU(n), JHEP 10 (2004) 047 hep-th/0404112.

[14] J. Maldacena, G. W. Moore and N. Seiberg, Geometrical interpretation of D-branes in gauged WZW models, JHEP 07 (2001) 046 hep-th/0105038.

[15] T. Quella and V. Schomerus, Symmetry breaking boundary states and defect lines, JHEP 06 (2002) 028 hep-th/0203161.

[16] T. Quella, On the hierarchy of symmetry breaking D-branes in group manifolds, JHEP 12 (2002) 009 [hep-th/0209157].

[17] A. Recknagel, Permutation branes, JHEP 04 (2003) 041 hep-th/0208119.

[18] J. M. Figueroa-O'Farrill and S. Stanciu, D-branes in $A d S_{3} \times S^{3} \times S^{3} \times S^{1}$, JHEP 04 (2000) 005 hep-th/0001199.

[19] M. R. Gaberdiel and S. Schäfer-Nameki, D-branes in an asymmetric orbifold, Nucl. Phys. B654 (2003) 177-196 hep-th/0210137.

[20] G. Sarkissian and M. Zamaklar, Symmetry breaking, permutation D-branes on group manifolds: Boundary states and geometric description, Nucl. Phys. B696 (2004) 66-106 hep-th/0312215. 
[21] I. Brunner and M. R. Gaberdiel, Matrix factorisations and permutation branes, JHEP 07 (2005) 012 hep-th/0503207].

[22] E. Witten, Nonabelian bosonization in two dimensions, Commun. Math. Phys. 92 (1984) 455-472.

[23] D. Gepner and E. Witten, String theory on group manifolds, Nucl. Phys. B278 (1986) 493.

[24] A. Yu. Alekseev and V. Schomerus, D-branes in the WZW model, Phys. Rev. D60 (1999) 061901 hep-th/9812193.

[25] K. Gawedzki, Conformal field theory: A case study, hep-th/9904145.

[26] G. Felder, J. Fröhlich, J. Fuchs and C. Schweigert, The geometry of WZW branes, J. Geom. Phys. 34 (2000) 162-190 hep-th/9909030.

[27] P. Bordalo, S. Ribault and C. Schweigert, Flux stabilization in compact groups, JHEP 10 (2001) 036 hep-th/0108201.

[28] C. Klimcik and P. Severa, Open strings and D-branes in WZNW models, Nucl. Phys. B488 (1997) 653-676 hep-th/9609112.

[29] C. Bachas, M. R. Douglas and C. Schweigert, Flux stabilization of D-branes, JHEP 05 (2000) 048 hep-th/0003037].

[30] E. S. Fradkin and A. A. Tseytlin, Nonlinear electrodynamics from quantized strings, Phys. Lett. B163 (1985) 123.

[31] A. Abouelsaood, C. G. Callan, C. R. Nappi and S. A. Yost, Open strings in background gauge fields, Nucl. Phys. B280 (1987) 599.

[32] C. G. Callan, C. Lovelace, C. R. Nappi and S. A. Yost, String loop corrections to beta functions, Nucl. Phys. B288 (1987) 525.

[33] S. Ribault, D3-branes in NS5-branes backgrounds, JHEP 02 (2003) 044 hep-th/0301092.

[34] I. Affleck and A. W. W. Ludwig, Universal noninteger "ground state degeneracy" in critical quantum systems, Phys. Rev. Lett. 67 (1991) 161-164.

[35] J. A. Harvey, S. Kachru, G. W. Moore and E. Silverstein, Tension is dimension, JHEP 03 (2000) 001 hep-th/9909072.

[36] N. Seiberg and E. Witten, String theory and noncommutative geometry, JHEP 09 (1999) 032 hep-th/9908142]. 
[37] A. Yu. Alekseev, S. Fredenhagen, T. Quella and V. Schomerus, Non-commutative gauge theory of twisted D-branes, Nucl. Phys. B646 (2002) 127-157 hep-th/0205123.

[38] J. Maldacena, G. W. Moore and N. Seiberg, D-brane instantons and K-theory charges, JHEP 11 (2001) 062 hep-th/0108100.

[39] S. Fredenhagen and C. Restuccia, DBI analysis of generalised permutation branes, arXiv:0908.1049 [hep-th].

[40] A. Giveon and E. Kiritsis, Axial vector duality as a gauge symmetry and topology change in string theory, Nucl. Phys. B411 (1994) 487-508 hep-th/9303016].

[41] S. Förste, D-branes on a deformation of SU(2), JHEP 02 (2002) 022 hep-th/0112193.

[42] C. Caviezel, S. Fredenhagen and M. R. Gaberdiel, The RR charges of A-type Gepner models, JHEP 01 (2006) 111 hep-th/0511078.

[43] M. Oshikawa and I. Affleck, Boundary conformal field theory approach to the critical two-dimensional Ising model with a defect line, Nucl. Phys. B495 (1997) 533-582 cond-mat/9612187.

[44] P. A. Pearce, L. Chim and C. Ahn, Excited TBA equations II: Massless flow from tricritical to critical Ising model, Nucl. Phys. B660 (2003) 579-606 [hep-th/0302093.

[45] M. R. Gaberdiel and A. Recknagel, Conformal boundary states for free bosons and fermions, JHEP 11 (2001) 016 hep-th/0108238.

[46] R. A. Janik, Exceptional boundary states at $c=1$, Nucl. Phys. B618 (2001) 675-688 hep-th/0109021.

[47] A. Cappelli and G. D'Appollonio, Boundary states of $c=1$ and $3 / 2$ rational conformal field theories, JHEP 02 (2002) 039 hep-th/0201173.

[48] M. R. Gaberdiel and H. Klemm, $N=2$ superconformal boundary states for free bosons and fermions, Nucl. Phys. B693 (2004) 281-301 hep-th/0404062.

[49] B. Blackadar, K-theory for operator algebras. Springer, 1986. 